\title{
THE ATTRACTOR OF RENORMALIZATION AND RIGIDITY OF TOWERS OF CRITICAL CIRCLE MAPS
}

\author{
MICHAEL YAMPOLSKY
}

\begin{abstract}
We demonstrate the existence of a global attractor $\mathcal{A}$ with a Cantor set structure for the renormalization of critical circle mappings. The set $\mathcal{A}$ is invariant under a generalized renormalization transformation, whose action on $\mathcal{A}$ is conjugate to the twosided shift.
\end{abstract}

\section{INTRODUCTION}

The empirical discovery of universality phenomena in the transition to chaos in onedimensional dynamical systems during the late 1970's made a great impact on the subject. To explain these phenomena, parallels with statistical physics were drawn, and Onedimensional Renormalization Theory was born from this effort. The main object of this theory is a renormalization transformation acting on an appropriate class of dynamical systems, and the universality phenomena are related to the hyperbolic dynamics of the transformation.

The first renormalization transformation was constructed by Feigenbaum, and Coullet \& Tresser in the setting of unimodal maps. Unimodal renormalization theory enjoyed spectacular progress since Sullivan introduced into it the methods of complex-analytic dynamics [Sul1]. The works of Sullivan [Sul2, MvS], McMullen [McM2] and Lyubich [Lyu3, Lyu4] culminated in establishing the hyperbolicity of the unimodal renormalization operator, thus providing the mathematical basis for the universality phenomena in unimodal dynamics.

The theory of renormalization of critical circle maps has developed alongside with the unimodal theory. Its objects, the critical circle maps, are orientation preserving selfhomeomorphisms of the circle $\mathbf{T}=\mathbb{R} / \mathbb{Z}$ of class $C^{3}$ with a single critical point $c$. A further assumption is made that the critical point is of cubic type. This means that for a lift $\bar{f}: \mathbb{R} \rightarrow \mathbb{R}$ of a critical circle map $f$ with critical points at integer translates of $\bar{c}$,

$$
\bar{f}(x)-\bar{f}(\bar{c})=(x-\bar{c})^{3}(\text { const }+O(x-\bar{c})) .
$$

Examples of analytic critical circle maps are provided by the projections $f_{\theta}$ to $\mathbb{R} / \mathbb{Z}$ of homeomorphisms of the standard (or Arnold's) family

$$
A_{\theta}(x)=x+\theta-\frac{1}{2 \pi} \sin 2 \pi x .
$$

The rotation number of a critical circle map $f$ will be denoted by $\rho(f)$. The number theoretical properties of $\rho(f)$ have dynamical implications for $f$. In particular, by a theorem of Yoccoz \Yod, in the case when $\rho(f)$ is irrational $f$ is topologically conjugate to the rigid 
rotation of the circle by angle $\rho(f)$. It is useful to associate to a rotation number a continued fraction expansion

$$
\rho(f)=\frac{1}{r_{0}+\frac{1}{r_{1}+\frac{1}{r_{2}+\cdots}}}
$$

which we will further abbreviate as $\rho(f)=\left[r_{0}, r_{1}, \ldots\right]$ for typographical convenience. The sequence $\left\{r_{i}\right\}$ is infinite if and only if $\rho(f)$ is irrational, in this case it is said to be of type bounded by $B$ if all terms $r_{i}$ are not greater than $B$.

The renormalization operator $\mathcal{R}$ for critical circle maps is defined in the language of critical commuting pairs. This approach first appeared in ORSS and, in a slightly different form, in [FKS], and has been further developed by Lanford [Lan1, Lan2] and others. Critical commuting pairs correspond to smooth conjugacy classes of critical circle maps; in particular, they posess rotation numbers, on which renormalization acts as the Gauss shift $G: \rho \rightarrow\{1 / \rho\}$. All pairs with non-zero rotation numbers are renormalizable, those with irrational rotation numbers are infinitely renormalizable. The main open question of the theory is the Hyperbolicity Conjecture, which postulates the existence of a smooth structure on the space of commuting pairs in which the renormalization transformation $\mathcal{R}$ is globally hyperbolic with one-dimensional expanding direction. In its full generality the Conjecture is due to Lanford (see [Lan2]), below we discuss it in some more detail. Although the first attempts to prove the Conjecture were confined to the framework of onedimensional smooth dynamics, the importance of analytic methods was early understood. In 1986 Eckmann and H. Epstein EE constructed a class of real-analytic maps invariant under $\mathcal{R}$, the so-called Epstein class $\mathcal{E}$. It was further shown (see [dF1]) that renormalizations of $C^{3}$ circle maps converge to $\mathcal{E}$, by a recent result of de Faria and de Melo dFdM1] the convergence occurs at a geometric rate.

In dF1, dF2 de Faria has adapted Sullivan's Renormalization Theory to the setting of critical circle mappings. De Faria has defined holomorphic extensions of renormalizations of maps in the Epstein class, which are the appropriate analogues of quadratic-like maps. Using Sullivan's techniques, he then demonstrated the existence of complex a priori bounds for such extensions in the case of maps of bounded type, and obtained the following renormalization convergence result:

Theorem [aF1, $\mathrm{dF} 2]$. Let $f_{1}$ and $f_{2}$ be two critical circle maps in $\mathcal{E}$ with the same rotation numbers in $\mathbb{R} \backslash \mathbb{Q}$ of bounded type. Then

$$
\operatorname{dist}_{C^{r}}\left(\mathcal{R}^{\circ n} f_{1}, \mathcal{R}^{\circ n} f_{2}\right) \rightarrow 0 \text { for all } 0 \leq r<\infty
$$

As a consequence, for any $B \geq 1$ there exists a closed $\mathcal{R}$-invariant set $\mathcal{A}_{B}$ of critical commuting pairs such that for any $f \in \mathcal{E}$ whose type is bounded by $B, \mathcal{R}^{\circ n} f \rightarrow \mathcal{A}_{B}$. In a recent work de Faria and de Melo dadM2 employed McMullen's towers techniques to demonstrate that the convergence to the attractor $\mathcal{A}_{B}$ happens at a geometric rate. 
In Ya1 we establish the existence of complex a priori bounds for circle maps with an arbitrary irrational rotation number, using methods developed in [LY]. This enabled us to extend the above stated renormalization convergence result of de Faria to circle maps with arbitrary irrational rotation numbers, getting rid of the condition of bounded type.

In this paper we establish the existence of a global attractor $\mathcal{A}$ for the renormalization operator $\mathcal{R}$, with a "horseshoe" structure. Let $\Sigma$ be the space of bi-infinite sequence of natural numbers, and denote by $\sigma: \Sigma \rightarrow \Sigma$ the shift on this space:

$$
\sigma:\left(r_{i}\right)_{-\infty}^{\infty} \mapsto\left(r_{i+1}\right)_{-\infty}^{\infty}
$$

For future use let us complement the natural numbers with the symbol $\infty$, and denote by $\bar{\Sigma}$ the space $(\mathbb{N} \cup\{\infty\})^{\mathbb{Z}}$. We prove the following:

Theorem A. There exists a $\mathcal{R}$-invariant set $\mathcal{I}$ of commuting pairs with irrational rotation numbers with the following properties. The action of $\mathcal{R}$ on $\mathcal{I}$ is bijective. Moreover, there is a one-to-one correspondence

$$
i: \mathcal{I} \rightarrow \Sigma
$$

such that if $\zeta=i^{-1}\left(\ldots, r_{-k}, \ldots, r_{-1}, r_{0}, r_{1}, \ldots, r_{k}, \ldots\right)$ then $\rho(\zeta)=\left[r_{0}, r_{1}, \ldots, r_{k}, \ldots\right]$ and thus the action of $\mathcal{R}$ on $\mathcal{I}$ is conjugate to the shift:

$$
i \circ \mathcal{R} \circ i^{-1}=\sigma .
$$

The set $\mathcal{I}$ is pre-compact in Carathéodory topology (see $\S 2$ for the definiton), its closure $\mathcal{A}$ is the attractor for the renormalization operator:

$$
\mathcal{R}^{\circ n} \zeta \rightarrow \mathcal{A}, \text { for all } \zeta \in \mathcal{E} \text { with } \rho(\zeta) \in \mathbb{R} \backslash \mathbb{Q},
$$

where the convergence is understood in the sense of Carathéodory topology (it implies, in particular, that the analytic extensions of the renormalized pairs converge uniformly on compact sets). More precisely, for any pair $\zeta^{\prime} \in \mathcal{A}$ with $\rho(\zeta)=\rho\left(\zeta^{\prime}\right)$ we have

$$
\operatorname{dist}\left(\mathcal{R}^{\circ n} \zeta, \mathcal{R}^{\circ n} \zeta^{\prime}\right) \rightarrow 0
$$

for the $C^{0}$-distance between the analytic extensions of the renormalized pairs on an open neighborghood of the origin.

The proof of the above theorem is inspired by the argument of McMullen for the convergence of unimodal renormalizations [McM2]. Following [McM2] we consider the geometric limits $T$ of various rescalings of sequences of renormalizations $\left(\zeta, \mathcal{R} \zeta, \ldots, \mathcal{R}^{\circ n} \zeta\right)$ of increasing lengths. The objects $T$ should be thought of as bi-infinite towers of nested dynamical systems in the plane. The proof of the theorem relies on the following uniqueness statement:

Tower Rigidity Theorem. There exists a unique (up to a homothety) bi-infinite tower for each bi-infinite sequence in $\bar{\Sigma}$.

We point out that a parallel statement has been proved by Hinkle Hin in the setting of unimodal maps with essentially bounded combinatorics. An important difference with the situation considered by McMullen and its adaptation to the case of circle maps carried 
out in dFdM2 is the presense of parabolic commuting pairs in a limiting tower. Forwardinfinite towers with parabolic elements which occur as geometric limits in a broad class of complex-analytic dynamical systems were considered in the dissertation of A. Epstein Ep1], who proved a rigidity theorem for such objects. It is conceivable that the constructions could be modified appropriately so that the results of Epstein could be applied directly in the setting of critical circle maps; we use some ergodic arguments to replace them.

The above mentioned parabolic elements of the attractor $\mathcal{A}$ are the pairs $\zeta$ in the closure of $\mathcal{I}$ with $\rho(\zeta)=0$ (we shall see below that such pairs posess fixed points with unit eigenvalues); on these pairs the action of the renormalization operator $\mathcal{R}$ is not defined. There is, however, a natural extension of $\mathcal{R}$ to commuting pairs with zero rotation number, the parabolic renormalization $\mathcal{P}$. Combining the action of $\mathcal{R}$ and $\mathcal{P}$ into the generalized renormalization operator $\mathcal{G}$ we obtain:

Theorem B. The attractor $\mathcal{A}$ is invariant under $\mathcal{G}$. The correspondence $i: \mathcal{I} \rightarrow \Sigma$ bijectively extends to $i: \mathcal{A} \rightarrow \bar{\Sigma}$ with the same properties, so that $i \circ \mathcal{G} \circ i^{-1}=\sigma$.

There is, strictly speaking, no canonical way to extend the parabolic renormalization operator $\mathcal{P}$ to Epstein commuting pairs with zero rotation number wich are not contained in the attractor $\mathcal{A}$. The parabolic renormalization of a pair $\zeta$ is determined by the selection of the rotation number of $\mathcal{P} \zeta$. We may, however, arbitrarily associate a rotation number with every $\zeta \in \mathcal{E}$ having $\rho(\zeta)=0$ and a parabolic fixed point, and in this way extend the generalized renormalization operator $\mathcal{G}$ to the parabolic elements of $\mathcal{E}$. For any such extension we will have the same convergence property:

Theorem C. The generalized renormalizations $\mathcal{G}^{n} \zeta$ converge to $\mathcal{A}$ for any parabolic pair $\zeta \in \mathcal{E}$.

As a consequence of the above theorems we obtain the following analogue of the golden mean renormalization fixed point:

Theorem D. There exists a commuting pair $\zeta_{0} \in \mathcal{A}$ such that for all maps $f$ with $\rho(f) \in$ $\mathbb{R} \backslash \mathbb{Q}$ and $G^{\circ n}(\rho(f)) \rightarrow 0$ we have

$$
\mathcal{R}^{\circ n} f \rightarrow \zeta_{0}
$$

Moreover, let $\mathcal{E}^{0} \subset \mathcal{E}$ be the set of Epstein pairs with zero rotation numbers, and let $\mathcal{P}^{0}: \mathcal{E}^{0} \rightarrow \mathcal{E}^{0}$ denote the appropriate parabolic renormalization operator. Then $\zeta_{0}$ is fixed under parabolic renormalization, $\mathcal{P}^{0} \zeta_{0}=\zeta_{0}$, and

$$
\mathcal{P}^{0} \zeta \rightarrow \zeta_{0} \text { for all } \zeta \in \mathcal{E}^{0}
$$

Acknowledgements. I would like to express my gratitude to A. Epstein and B. Hinkle, the discussions with them have greatly assisted me in producing this paper. Ben Hinkle has a parallel work Hin on unimodal maps with essentially bounded combinatorics, and I appreciate his willingness to let me consult his manuscript before it was finished. I wish to thank O. Lanford, whose numerous helpful comments on an earlier version of this paper helped me to streamline some of the arguments and to improve the exposition. I also would 
like to thank E. deFaria, who graciously provided me with preliminary copies of his papers with W. deMelo. Finally, my thanks go to Mikhail Lyubich for numerous stimulating conversations and constant moral support.

\section{PRELiminaries}

Some notations. The notation $D_{r}(z)$ will stand for the Euclidean disk with center $z \in \mathbb{C}$ and radius $r$. The unit disk $D_{1}(0)$ will be denoted $\mathbb{D}$. For two points $a$ and $b$ in the circle $\mathbf{T}$, $[a, b]$ will denote the shorter of the two arcs connecting them; $|[a, b]|$ will denote the length of the arc. For two points $a, b \in \mathbb{R},[a, b]$ will denote the closed interval with endpoints $a$, $b$ without specifying their order. The plane $(\mathbb{C} \backslash \mathbb{R}) \cup J$ with the parts of the real axis not contained in the interval $J \subset \mathbb{R}$ removed will be denoted $\mathbb{C}_{J}$.

We use dist and diam to denote the Euclidean distance and diameter in $\mathbb{C}$. We call two real numbers $x$ and $y K$-commensurable or simply commensurable if $K^{-1} \leq|x| /|y| \leq K$ for some $K>1$. Two sets $X$ and $Y$ in $\mathbb{C}$ are $K$-commensurable if their diameters are. In accordance with the established terminology, we shall say that a quantity is definite if it is greater than a universal positive constant. A set $B$ is contained well inside of a set $A \subset \mathbb{C}$ if $A \backslash B$ contains an annulus with definite modulus. Similarly, an interval $I \subset \mathbb{R}$ is contained well inside of another interval $J$ there exists a universal constant $K>0$ such that for each component $L$ of $J \backslash I$ we have $|L|>K|I|$.

Commuting pairs and renormalization of critical circle maps. Consider a critical circle mapping $f$ with a rotation number $\rho$, and let

$$
\rho(f)=\left[r_{0}, r_{1}, r_{2}, \ldots\right]
$$

be its (possibly finite) continued fraction expansion. We shall always assume that the critical point of $f$ is at $0 \in \mathbb{R} / \mathbb{Z}$. An iterate $f^{k}(0)$ is called a closest return of the critical point if the arc $\left[0, f^{k}(0)\right]$ contains no other iterates $f^{i}(0)$ with $i<k$. Since a circle homeomorphism $f$ is semi-conjugated to the rigid rotation by angle $\rho(f)$ (see for example MvS), the moments of closest returns are determined by the number-theoretic properties of $\rho(f)$. Namely, the closest returns occur at iterates $\left\{f^{q_{m}}(0)\right\}$ where $q_{m}$ 's are given recursively by $q_{m+1}=r_{m} q_{m}+q_{m-1}, q_{0}=1, q_{1}=r_{0}$. Thus the number $q_{m}$ appears as the denominator of the truncated continued fraction expansion of $\rho$ of length $m-1$ in its reduced form:

$$
p_{m} / q_{m}=\left[r_{0}, r_{1}, \ldots, r_{m-1}\right] .
$$

Set $I_{m} \equiv\left[0, f^{q_{m}}(0)\right]$. As a consequence of Świastek-Herman real a priori bounds ([Sw1, $\left.\mathrm{He}\right]$ ), the intervals $I_{m}$ and $I_{m+1}$ are $K$-commensurable, with a universal constant $K$ provided $m$ is large enough.

The general strategy of defining a renormalization of a given dynamical system (we are following [Lyu2 here) is to select a piece of its phase space, rescale it to the "original" size, and then consider the first return map to this piece. Historically, for a circle map $f$ the union of arcs $A_{m}=I_{m} \cup I_{m+1}$ is chosen as the domain for the return map. The first return map $R_{m}: A_{m} \rightarrow A_{m}$ is defined piecewise by by $f^{q_{m}}$ on $I_{m+1}$ and by $f^{q_{m+1}}$ on $I_{m}$. 
To view $R_{m}$ as a critical circle map we may identify the neighborhoods of points $f^{q_{m}}(0)$ and $f^{q_{m+1}}(0)$ by the iterate $f^{q_{m+1}-q_{m}}$. This identification transforms the arc $A_{m}$ into a $C^{3}-$ smooth closed one-dimensional manifold $\tilde{A}_{m}, R_{m}$ projects to a smooth homeomorphism $\tilde{R}_{m}: \tilde{A}_{m} \rightarrow \tilde{A}_{m}$ with a critical point at 0 . However, the manifold $\tilde{A}_{m}$ does not posess a canonical affine structure; the choice of a smooth identification $\phi: \tilde{A}_{m} \rightarrow \mathbb{R} / \mathbb{Z}$ gives rise to a plethora of different critical circle maps, all smoothly conjugate. The above discussion illustrates why the space of critical circle maps is not suited for defining a renormalization transformation, and motivates the introduction of the following objects:

Definition 2.1. A commuting pair $\zeta=(\eta, \xi)$ consists of two smooth orientation preserving interval homeomorphisms $\eta: I_{\eta} \rightarrow \eta\left(I_{\eta}\right), \xi: I_{\xi} \rightarrow \xi\left(I_{\xi}\right)$, where

(I) $I_{\eta}=[0, \xi(0)], I_{\xi}=[\eta(0), 0]$;

(II) Both $\eta$ and $\xi$ have homeomorphic extensions to interval neighborhoods of their respective domains with the same degree of smoothness, which commute, $\eta \circ \xi=\xi \circ \eta$;

(III) $\xi \circ \eta(0) \in I_{\eta}$;

(IV) $\eta^{\prime}(x) \neq 0 \neq \xi^{\prime}(y)$, for all $x \in I_{\eta} \backslash\{0\}$, and all $y \in I_{\xi} \backslash\{0\}$.

Commuting pairs were first used to define the renormalization transformation by Ostlund, Rand, Sethna, and Siggia ORSS. Feigenbaum, Kadanoff, and Shenker FKS defined renormalization by means of a slightly different formalism.

A critical commuting pair is a commuting pair $(\eta, \xi)$ whose maps can be decomposed near zero as $\eta=h_{\eta} \circ Q \circ H_{\eta}$, and $\xi=h_{\xi} \circ Q \circ H_{\xi}$, where $h_{\eta}, h_{\xi}, H_{\eta}, H_{\xi}$ are real analytic diffeomorphisms and $Q(x)=x^{3}$. We shall further require a technical assumption that $\xi$ analytically extends to an interval $(a, b) \ni 0$ with $\xi(a, b) \supset[\eta(0), \xi(0)]$, and has a single critical point 0 in this interval. The space of critical commuting pairs modulo affine conjugacy endowed with $C^{0}$ topology, will be denoted by $\boldsymbol{C}$.

Let $f$ be a critical circle mapping, whose rotation number $\rho$ has a continued fraction expansion (2.1) with at least $m+1$ terms. Let $p_{m}$ and $q_{m}$ be as in (2.2). The pair of iterates $f^{q_{m+1}}$ and $f^{q_{m}}$ restricted to the circle arcs $I_{m}$ and $I_{m+1}$ correspondingly can be viewed as a critical commuting pair in the following way. Let $\bar{f}$ be the lift of $f$ to the real line satisfying $\bar{f}^{\prime}(0)=0$, and $0<\bar{f}(0)<1$. For each $m>0$ let $\bar{I}_{m} \subset \mathbb{R}$ denote the closed interval adjacent to zero which projects down to the interval $I_{m}$. Let $\tau: \mathbb{R} \rightarrow \mathbb{R}$ denote the translation $x \mapsto x+1$. Let $\eta: \bar{I}_{m} \rightarrow \mathbb{R}, \xi: \bar{I}_{m+1} \rightarrow \mathbb{R}$ be given by $\eta \equiv \tau^{-p_{m+1}} \circ \bar{f}^{q_{m+1}}$, $\xi \equiv \tau^{-p_{m}} \circ \bar{f}^{q_{m}}$. Then the pair of maps $\left(\eta\left|\bar{I}_{m}, \xi\right| \bar{I}_{m+1}\right)$ forms a critical commuting pair corresponding to $\left(f^{q_{m+1}}\left|I_{m}, f^{q_{m}}\right| I_{m+1}\right)$. Henceforth we shall simply denote this commuting pair by

$$
\left(f^{q_{m+1}}\left|I_{m}, f^{q_{m}}\right| I_{m+1}\right) .
$$

This allows us to readily identify the dynamics of the above commuting pair with that of the underlying circle map, at the cost of a minor abuse of notation.

Following dFdM1, we say that the height $\chi(\zeta)$ of a critical commuting pair $\zeta=(\eta, \xi)$ is equal to $r$, if

$$
0 \in\left[\eta^{r}(\xi(0)), \eta^{r+1}(\xi(0))\right]
$$


If no such $r$ exists, we set $\chi(\zeta)=\infty$, in this case the map $\eta \mid I_{\eta}$ has a fixed point. For a pair $\zeta$ with $\chi(\zeta)=r<\infty$ one verifies directly that the mappings $\eta \mid\left[0, \eta^{r}(\xi(0))\right]$ and $\eta^{r} \circ \xi \mid I_{\xi}$ again form a commuting pair. For a commuting pair $\zeta=(\eta, \xi)$ we will denote by $\widetilde{\zeta}$ the pair $\left(\widetilde{\eta}\left|\widetilde{I}_{\eta}, \widetilde{\xi}\right| \widetilde{I}_{\xi}\right)$ where tilde means rescaling by a linear factor $\lambda=\frac{1}{\left|I_{\eta}\right|}$.

Definition 2.2. The renormalization of a real commuting pair $\zeta=(\eta, \xi)$ is the commuting pair

$$
\mathcal{R} \zeta=\left(\widetilde{\eta^{r} \circ \xi}\left|\widetilde{I}_{\xi}, \widetilde{\eta}\right|\left[0, \widetilde{\eta^{r}(\xi(0))}\right]\right) .
$$

The non-rescaled pair $\left(\eta^{r} \circ \xi\left|I_{\xi}, \eta\right|\left[0, \eta^{r}(\xi(0))\right]\right)$ will be referred to as the pre-renormalization $p \mathcal{R} \zeta$ of the commuting pair $\zeta=(\eta, \xi)$.

For a pair $\zeta$ we define its rotation number $\rho(\zeta) \in[0,1]$ to be equal to the continued fraction $\left[r_{0}, r_{1}, \ldots\right]$ where $r_{i}=\chi\left(\mathcal{R}^{i} \zeta\right)$. In this definition $1 / \infty$ is understood as 0 , hence a rotation number is rational if and only if only finitely many renormalizations of $\zeta$ are defined; if $\chi(\zeta)=\infty, \rho(\zeta)=0$. Thus defined, the rotation number of a commuting pair can be viewed as a rotation number in the usual sense via the following construction. Given a critical commuting pair $\zeta=(\eta, \xi)$ we can regard the interval $I=[\eta(0), \xi \circ \eta(0)]$ as a circle, identifying $\eta(0)$ and $\xi \circ \eta(0)$ and define $f_{\zeta}: I \rightarrow I$ by

$$
f_{\zeta}=\left\{\begin{array}{l}
\xi \circ \eta(x) \text { for } x \in[\eta(0), 0] \\
\eta(x) \text { for } x \in[0, \xi \circ \eta(0)]
\end{array}\right.
$$

We perform glueing together of $\eta(0)$ to $\xi \circ \eta(0)$ by the mapping $\xi$, which by the condition (II) above extends to a smooth homeomorphism of open neighborhoods. The quotient of the interval $I$ is a closed one-dimensional manifold $M$, the mapping $f_{\zeta}$ projects down to a smooth homeomorphism $F_{\zeta}: M \rightarrow M$. Identifying $M$ with the circle by a diffeomorphism $\phi: M \rightarrow S^{1}$ we recover a critical circle mapping $f^{\phi}=\phi \circ F_{\zeta} \circ \phi^{-1}$. The critical circle mappings corresponding to two different choices of $\phi$ are conjugated by a diffeomorphism, and thus we recovered a smooth conjugacy class of circle mappings from a critical commuting pair. It is immediately seen, that:

Proposition 2.1. The rotation number of mappings in the above constructed conjugacy class is equal to $\rho(\zeta)$.

The advantage of defining $\rho(\zeta)$ using a sequence of heights is in having a way of distinguishing the commuting pairs with rotation numbers 0 and 1 , this way we also remove the ambiguity in prescribing a continued fraction expansion to rational rotation numbers.

For $\rho=\left[r_{0}, r_{1}, \ldots\right] \in[0,1]$ let us set

$$
G(\rho)=\left[r_{1}, r_{2}, \ldots\right]=\left\{\frac{1}{\rho}\right\},
$$

where $\{x\}$ denotes the fractional part of a real number $x(G$ is usually referred to as the Gauss map). As follows from the definition,

$$
\rho(\mathcal{R} \zeta)=G(\rho(\zeta))
$$


for a real commuting pair $\zeta$ with $\rho(\zeta) \neq 0$.

The renormalization of the real commuting pair (2.3), associated to some critical circle map $f$, is the rescaled pair $\left(\widetilde{f^{q_{m+2}}}\left|\widetilde{I_{m+1}}, \widetilde{f^{q_{m+1}}}\right| \widetilde{I_{m+2}}\right)$. Thus for a given critical circle map $f$ the renormalization operator recovers the (rescaled) sequence of the first return maps:

$$
\left\{\left(\widetilde{f^{q_{i+1}}}\left|\widetilde{I}_{i}, \widetilde{f^{q_{i}}}\right| \widetilde{I_{i+1}}\right)\right\}_{i=1}^{\infty} .
$$

Let us denote by $\boldsymbol{C}_{\infty} \subset \boldsymbol{C}$ the space of critical commuting pairs $\zeta$ modulo rescaling with $\chi(\zeta)=\infty$. It is clear that $\mathcal{R}: \boldsymbol{C} \backslash \boldsymbol{C}_{\infty} \rightarrow \boldsymbol{C}$. Let us note:

Remark 2.1. The map $\mathcal{R}: \boldsymbol{C} \backslash \boldsymbol{C}_{\infty} \rightarrow \boldsymbol{C}$ is injective.

Proof. Let $\zeta=(\eta, \xi)$ be a pair in $\mathcal{R}\left(\boldsymbol{C} \backslash \boldsymbol{C}_{\infty}\right)$. For $r \in \mathbb{N}$ let $\gamma_{r}$ be the maximal analytic extension of $\xi^{-r} \circ \eta$. Choose the smallest $r_{0}$ for which $\gamma_{r_{0}}^{-1}$ is differentiable at $\xi(0)$. Setting $\gamma=\gamma_{r_{0}}$ and

$$
\zeta_{-1}=\left(\left.\xi\right|_{[0, \gamma(0)]},\left.\gamma\right|_{[0, \xi(0)]}\right)
$$

we have $\zeta_{-1} \in \boldsymbol{C}$ and $\mathcal{R} \zeta_{-1}=\zeta$.

Renormalization hyperbolicity conjecture. We would like to discuss briefly the general Renormalization Hyperbolicity conjecture and its connection to the existence of the attractor of the renormalization operator.

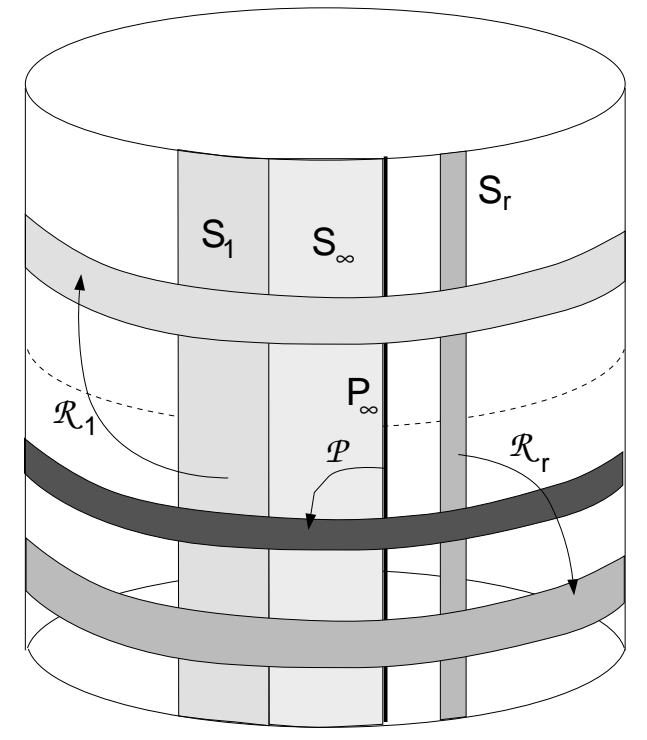

FIGURE 1.

Renormalization Hyperbolicity Conjecture. There exists a renormalization-invariant set of critical commuting pairs with the structure of an infinite dimensional smooth manifold, with respect to which the renormalization transformation $\mathcal{R}$ is globally uniformly hyperbolic, with one-dimensional expanding direction. 
Moreover, if $t$ denotes the expanded coordinate in a local chart, then the dependence $t \mapsto \rho(\zeta(t, \cdot))$ is continuous and (not strictly) monotone.

In this generality the conjecture is due to Lanford [Lan2, it remains the main open question of the theory. To illustrate the conjecture it is convenient to consider the following caricature. Let $\rho=\phi(\theta): \mathbf{T} \rightarrow \mathbf{T}$ be a monotone continuous function such that $\phi^{-1}(\rho)$ is a point for $\rho$ irrational and an interval otherwise. An example of such a function is the dependence $\theta \mapsto \rho\left(f_{\theta}\right)$ of the rotation number of a standard map on the parameter. Imagine the relevant space of commuting pairs as an infinite-dimensional cylinder $\mathrm{C}=\mathbf{T} \times \mathrm{C}^{\prime}$, where the rotation number of a commuting pair $\zeta(\theta, \cdot)$ with the equatorial coordinate $\theta \in \mathbf{T}$ is $\rho(\theta)$. The cylinder $\mathrm{C}$ is partitioned into strips (cf. Figure 1)

$$
\mathrm{S}_{r}=\left\{\zeta \in \mathrm{C} \mid \rho(\zeta)=\left[r, r_{1}, \ldots\right]\right\} \text { for } r=1, \ldots, \infty
$$

A boundary component of the strip $\mathrm{S}_{\infty}$ is the hypersurface $\mathrm{P}_{\infty} \subset \mathrm{S}_{\infty}$ with the property that a pair $\zeta \in \mathrm{P}_{\infty}$ if and only if it has a fixed point with unit eigenvalue. The sets $\mathrm{S}_{r}$ accumulate on $\mathrm{P}_{\infty}$ in clockwise direction.

It is natural to think of the transformation $\mathcal{R}: \mathrm{C} \backslash \mathrm{S}_{\infty} \rightarrow \mathrm{C}$ as being defined piecewise, given on each $\mathrm{S}_{r}, r \neq \infty$ by the formula:

$$
\mathcal{R}_{r}:(\eta, \xi) \mapsto\left(\eta, \eta^{r} \circ \xi\right) .
$$

The operator $\mathcal{R}_{r}$ uniformly expands the strip $\mathrm{S}_{r}$ in the equatorial direction, and uniformly contracts it in all other directions, mapping it onto a thin cylinder intersecting all the strips. The invariant set $\mathcal{I}$ is seen in this picture as intersections of the "boxes"

$\mathcal{R}_{r_{-1}}\left(\mathrm{~S}_{r_{-1}} \cap \mathcal{R}_{r_{-2}}\left(\mathrm{~S}_{r_{-2}} \cap \cdots\left(\mathcal{R}_{r_{-n}} \mathrm{~S}_{r_{-n}}\right) \cdots\right) \cap \mathrm{S}_{r_{0}} \cap \mathcal{R}_{r_{0}}^{-1}\left(\mathrm{~S}_{r_{1}} \cap \mathcal{R}_{r_{1}}^{-1}\left(\mathrm{~S}_{r_{2}} \cap \cdots \mathcal{R}_{r_{n-1}}^{-1}\left(\mathrm{~S}_{r_{n}}\right) \cdots\right)\right.\right.$

The parabolic renormalization $\mathcal{P}$ which we define below transforms the set $\mathcal{P}_{\infty}$ into a thin equatorial cylinder, the hyperbolicity conjecture can be extended in the obvious way to include this transformation.

Holomorphic commuting pairs. Following [dF1, dF2 we say that a real commuting

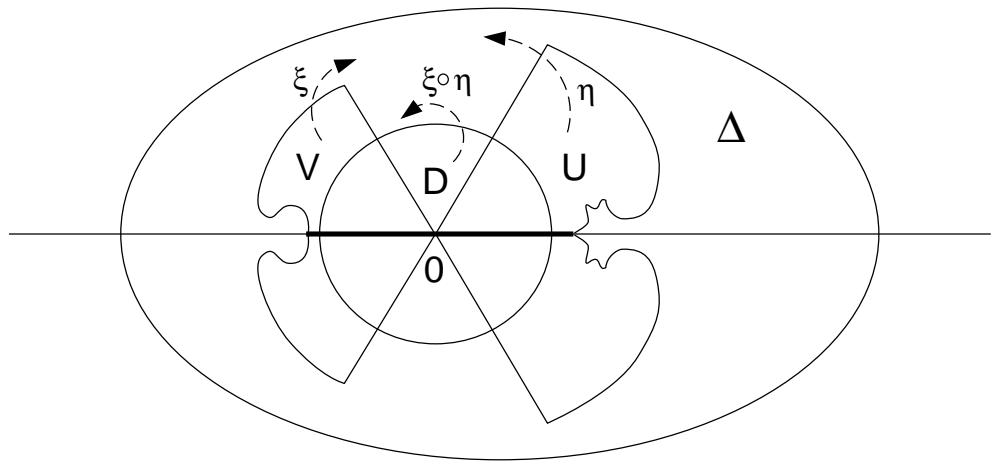

FiguRE 2.

pair $(\eta, \xi)$ extends to a holomorphic commuting pair $\mathcal{H}$ (cf. Figure 2 ) if there exist four $\mathbb{R}$-symmetric domains $\Delta, D, U, V$, and a holomorphic mapping $\nu$, such that 
- $\bar{D}, \bar{U}, \bar{V} \subset \Delta, \bar{U} \cap \bar{V}=\{0\} ; U \backslash D, V \backslash D, D \backslash U$, and $D \backslash V$ are nonempty connected sets, $U \supset I_{\eta}, V \supset I_{\xi}$;

- mappings $\eta: U \rightarrow \Delta \cap \mathbb{C}_{\eta\left(J_{U}\right)}$ and $\xi: V \rightarrow \Delta \cap \mathbb{C}_{\xi\left(J_{V}\right)}$ are onto and univalent, where $J_{U}=U \cap \mathbb{R}, J_{V}=V \cap \mathbb{R}$

- $\nu: D \rightarrow \Delta \cap \mathbb{C}_{\nu\left(J_{D}\right)}$ is a three-fold branched covering with a unique fixed point at zero, where $J_{D}=D \cap \mathbb{R}$;

- $\eta$ and $\xi$ have holomorphic extensions to a certain neighborhood of the origin where both $\eta \circ \xi$ and $\xi \circ \eta$ are defined, and $\eta \circ \xi(z)=\xi \circ \eta(z)=\nu(z)$ for all $z$ in that neighborhood.

As the following proposition shows, the mapping $\nu$ is nothing else, but the composition of $\eta$ and $\xi$ :

Proposition 2.2 (Proposition II.1, $\mathrm{dF2}$ ). Under the above conditions, the mappings $\eta$ and $\xi$ have analytic extensions to $U \cup D$ and $V \cup D$ correspondingly. Moreover, $\eta: D \rightarrow$ $V \cap \mathbb{C}_{\left[\xi^{-1}(\eta(0)), 0\right]}$ and $\xi: D \rightarrow U$ are three-fold branched covering maps, and $\nu=\eta \circ \xi$.

Set $\Omega=D \cup U \cup V$. One immediately observes that if a commuting pair $\zeta=(\eta, \xi)$ with a finite height has a holomorphic pair extension $\mathcal{H}: \Omega \rightarrow \Delta$, then there exists a holomorphic commuting pair $\mathcal{G}: \Omega^{\prime} \rightarrow \Delta$ whose restriction coincides with $\mathcal{R} \zeta$. We shall refer to the commuting pair $\zeta$ as underlying $\mathcal{H}$, and write $\zeta=\mathcal{H} \cap \mathbb{R}$.

We say that a real commuting pair $(\eta, \xi)$ with an irrational rotation number has complex bounds, if all its renormalizations extend to holomorphic commuting pairs with definite moduli, that is

$$
\bmod (\Delta \backslash \Omega)>\mu>0 \text {. }
$$

The shadow of the holomorphic commuting pair is the piecewise holomorphic mapping $S_{\mathcal{H}}: \Omega \rightarrow \Delta$, given by

$$
S_{\mathcal{H}}(z)=\left\{\begin{array}{l}
\eta(z), z \in U \\
\xi(z), z \in V \\
\xi \circ \eta(z), z \in D \backslash(U \cup V)
\end{array}\right.
$$

The shadow of a holomorphic pair captures its dynamics in the following sense:

Proposition 2.3 (Prop. II.4. [dF2]). Given a holomorphic commuting pair $\mathcal{H}$ as above, consider its shadow $S_{\mathcal{H}}$. Let $I=\Omega \cap \mathbb{R}$, and $X=I \cup S_{\mathcal{H}}^{-1}(I)$. Then:

- The restriction of $S_{\mathcal{H}}$ to $\Omega \backslash X$ is a regular three fold covering onto $\Delta \backslash \mathbb{R}$.

- The orbits of $S_{\mathcal{H}}$ and $\mathcal{H}$ coincide as sets.

We will say that two holomorphic commuting pairs $\mathcal{H}: \Omega_{\mathcal{H}} \rightarrow D_{\mathcal{H}}$ and $\mathcal{G}: \Omega_{\mathcal{G}} \rightarrow D_{\mathcal{G}}$ are conjugate if there is a homeomorphism $h: D_{\mathcal{G}} \rightarrow D_{\mathcal{H}}$ such that

$$
S_{\mathcal{G}}=h^{-1} \circ S_{\mathcal{H}} \circ h .
$$

We will usually write simply $\mathcal{G}=h^{-1} \circ \mathcal{H} \circ h$, meaning that $h$ conjugates the corresponding elements of the two holomorphic pairs. We define the filled Julia set $K(\mathcal{H})$ of a holomorphic commuting pair $\mathcal{H}$ as the closure of the collection of all points which do not escape $\Omega$ under 
iteration of $S_{\mathcal{H}}$. This set is clearly compact and connected, it is full by the Maximum principle. Its boundary is the Julia set of $\mathcal{H}$, denoted by $J(\mathcal{H})$.

Holomorphic commuting pairs in the standard family. For each $0 \leq \theta<1$ let $A_{\theta}$ be the entire mapping given by

$$
A_{\theta}(z)=z+\theta+\frac{1}{2 \pi} \sin (2 \pi z) .
$$

Since $A_{\theta} \circ T=T \circ A_{\theta}$, where $T$ is the unit translation $z \mapsto z+1$, each $A_{\theta}$ is a lift of a holomorphic self-mapping of the cylinder, $f_{\theta}: \mathbb{C} / \mathbb{Z} \cong \mathbb{C}^{*}$. As each $A_{\theta}$ is real-analytic and satisfies $A_{\theta}^{\prime}(x)>0$ for $x \in \mathbb{R} \backslash \mathbb{Z}$, the restriction $f_{\theta} \mid \mathbf{T}$ is a critical circle map, whose rotation number we will denote $\rho(\theta)$. These restrictions are usually referred to as the standard family (or Arnold family) of circle homeomorphisms. Elementary considerations of monotone dependence on parameter imply that $\theta \rightarrow \rho(\theta)$ is a continuous non-decreasing map of $\mathbf{T}$ onto itself. Whenever $t \in \mathbf{T}$ is irrational, $\rho^{-1}(t)$ is a single point. For $t=p / q$ the set $\rho^{-1}(t)$ is a closed interval, for every parameter value in this interval the homeomorphism $f_{\theta}$ has a period $q$ orbit. This orbit has eigenvalue one at the two endpoints of the interval.

De Faria (cf. [dF1, dF2]) demonstrated, using some explicit estimates on the growth of the standard maps, that renormalizations of maps $f_{\theta}$ can be extended to holomorphic commuting pairs. Before formulating his result, let us briefly describe the geometry of a mapping $A_{\theta}$. The preimage of the real axis under $A_{\theta}$ consists of the axis itself together with the family of analytic curves

$$
\Gamma_{ \pm}^{k}: \operatorname{Re}(z)=k \pm \frac{1}{2 \pi} \arccos \frac{-2 \pi|\operatorname{Im}(z)|}{\sinh (2 \pi \operatorname{Im}(z))}
$$

where $k \in \mathbb{Z}$. For each $k$ the curves $\Gamma_{ \pm}^{k}$ meet at the critical point $c_{k}=k$ and are both asymptotic to the vertical lines $\operatorname{Re}(z)=k \pm 1 / 4$. Note that each $c_{k}$ is of cubic type. The curves $\Gamma_{ \pm}^{k}$ and $\mathbb{R}$ partition the complex plane into simply-connected regions each of which is univalently mapped onto $\mathbb{H}$ or $-\mathbb{H}$ by $A_{\theta}$. Now denote by $U_{n}$ the connected component of the preimage $\left(A_{\theta}^{q_{n}}\right)^{-1}(\mathbb{H})$ whose boundary contains the point $T^{-p_{n+1}}\left(A_{\theta}^{q_{n+1}}(0)\right)$. The closure of the union of $U_{n}$ and its reflection in $x$-axis will be denoted $\hat{U}_{n}$. Similarly let $V_{n}$ be the component of $\left(A_{\theta}^{q_{n+1}}\right)^{-1}(\mathbb{H})$ with $\mathrm{cl} V_{n} \ni T^{-p_{n}}\left(A_{\theta}^{q_{n}}(0)\right)$, and set $\hat{V}_{n}$ to be the union of $\mathrm{cl} V_{n}$ with its vertical reflection.

Lemma 2.4 ( dF1, dF2). Set $\eta=T^{-p_{n+1}} \circ A_{\theta}^{q_{n+1}}$ and $\xi=T^{-p_{n}} \circ A_{\theta}^{q_{n}}$. For all sufficiently large $R$ the preimages $U_{n, R}=\eta^{-1}\left(D_{R}(0)\right) \cap \hat{U}_{n}$ and $V_{n, R}=\xi^{-1}\left(\left(D_{R}(0)\right) \cap \hat{V}_{n}\right)$ are compactly contained in $D_{R}(0)$. Thus the pair of maps $(\eta, \xi)$ extends to a holomorphic pair with range $D_{R}(0)$. Moreover the modulus of this holomorphic pair tends to infinity with $R$.

The significance of these examples in the Renormalization Theory is due to a rigidity property of standard maps:

Lemma 2.5 (see Lemma IV.8 dF2 ). Every real-symmetric holomorphic self-map of $\mathbb{C} / \mathbb{Z}$ which is topologically conjugate to a member of the family $\left\{f_{\theta}\right\}$ belongs to this family itself. 
Note, that $f_{\theta}$ is a finite-type analytic map of the cylinder, and thus, by a a theorem of L. Keen [Keen, does not have wandering domains. Combining Lemma 2.5 with the above described properties of the dependence $\theta \mapsto \rho(\theta)$ we have the following:

Lemma 2.6. Let $g$ be a real-symmetric self map of the cylinder $\mathbb{C} / \mathbb{Z}$ topologically conjugate to a map $f_{\theta}$. If $\rho(\theta)$ is irrational, or $f_{\theta}$ has a periodic orbit with eigenvalue one on the circle, then $g \equiv f_{\theta}$.

Taking further the first statement of the above Lemma, de Faria has shown:

Lemma 2.7 (dF1, dF2 ). If $\rho(\theta)$ is irrational, then $f_{\theta}$ admits no non-trivial, symmetric, invariant Beltrami differentials entirely supported on its Julia set.

The above properties of standard maps carry over to general holomorphic commuting pairs via quasiconformal straightening arguments.

A topology on a space of branched coverings. Consider the collection $\boldsymbol{B}$ of all triplets $(U, u, f)$, where $U \subset \mathbb{C}$ is a topological disk different from the whole plane, $u \in U$, and $f: U \rightarrow \mathbb{C}$ is a three-fold analytic branched covering map, with the only branch point at $u$. We will endow $\boldsymbol{B}$ with a topology as follows (cf. McM1).

Let $\left\{\left(U_{n}, u_{n}\right)\right\}$ be a sequence of open connected regions $U_{n} \subset \mathbb{C}$ with marked points $u_{n} \in U_{n}$. Recall that this sequence Carathéodory converges to a marked region $(U, u)$ if:

- $u_{n} \rightarrow u \in U$, and

- for any Hausdorff limit point $K$ of the sequence $\hat{\mathbb{C}} \backslash U_{n}, U$ is a component of $\hat{\mathbb{C}} \backslash K$.

For a simply connected $U \subset \mathbb{C}$ and $u \in U$ let $R_{(U, u)}: \mathbb{D} \rightarrow U$ denote the Riemann mapping with normalization $R_{(U, u)}(0)=u, R_{(U, u)}^{\prime}(0)>0$. By a classical result of Carathéodory, the Carathédory convergence of simply-connected regions $\left(U_{n}, u_{n}\right) \rightarrow(U, u)$ is equivalent to the locally uniform convergence of Riemann mappings $R_{\left(U_{n}, u_{n}\right)}$ to $R_{(U, u)}$.

For positive numbers $\epsilon_{1}, \epsilon_{2}, \epsilon_{3}$ and compact subsets $K_{1}$ and $K_{2}$ of the open unit disk $\mathbb{D}$, let the neighborhood $\mathcal{U}_{\epsilon_{1}, \epsilon_{2}, \epsilon_{3}, K_{1}, K_{2}}(U, u, f)$ of an element $(U, u, f) \in \boldsymbol{B}$ be the set of all $(V, v, g) \in \boldsymbol{B}$, for which:

- $|u-v|<\epsilon_{1}$

- $\sup _{z \in K_{1}}\left|R_{(V, v)}(z)-R_{(U, u)}(z)\right|<\epsilon_{2}$,

- and $R_{(U, u)}\left(K_{2}\right) \subset V$, and $\sup _{z \in R_{(U, u)}\left(K_{2}\right)}|f(z)-g(z)|<\epsilon_{3}$.

One verifies that the sets $\mathcal{U}_{\epsilon_{1}, \epsilon_{2}, \epsilon_{3}, K_{1}, K_{2}}(U, u, f)$ form a base of a topology on $\boldsymbol{B}$, which we will call Carathéodory topology. This topology is clearly Hausdorff, and the convergence of a sequence $\left(U_{n}, u_{n}, f_{n}\right)$ to $(U, u, f)$ is equivalent Carathéodory convergence of the marked regions $\left(U_{n}, u_{n}\right) \rightarrow(U, u)$ as well as locally uniform convergence $f_{n} \rightarrow f$.

Epstein class. An orientation preserving interval homeomorphism $g: I=[0, a] \rightarrow g(I)=$ $J$ belongs to the Epstein class $\mathcal{E}$ if it extends to an analytic three-fold branched covering map of a topological disk $G \supset I$ onto the double-slit plane $\mathbb{C}_{\tilde{J}}$, where $\tilde{J} \supset \mathrm{cl} J$. Any map 
$g$ in the Epstein class can be decomposed as

$$
g=Q_{c} \circ h
$$

where $Q_{c}(z)=z^{3}+c$, and $h: I \rightarrow[0, b]$ is a univalent map $h: G \rightarrow \Delta(h)$ onto the complex plane with six slits, which triple covers $\mathbb{C}_{\tilde{J}}$ under the cubic map $Q_{c}(z)$.

For any $s \in(0,1)$, let us introduce a smaller class $\mathcal{E}_{s} \subset \mathcal{E}$ of Epstein mappings $g: I=$ $[0, a] \rightarrow J \subset \tilde{J}$ for which both $|I|$ and $\operatorname{dist}(I, J)$ are $s^{-1}$-commensurable with $|J|$, the length of each component of $\tilde{J} \backslash J$ is at least $s|J|$, and $g^{\prime}(a)>s$. We will often refer to the space $\mathcal{E}$ as the Epstein class, and to each $\mathcal{E}_{s}$ as an Epstein class.

We say that a commuting pair $(\eta, \xi) \in \boldsymbol{C}$ belongs to the (an) Epstein class if both of its maps do. It immediately follows from the definitions that:

Lemma 2.8. The space of commuting pairs in the Epstein class $\mathcal{E}$ is invariant under renormalization.

We shall denote by $\boldsymbol{H}$ the space of holomorphic commuting pairs $\mathcal{H}: \Omega \rightarrow \Delta$ whose underlying real commuting pair $(\eta, \xi)$ is in the Epstein class. In this case both maps $\eta$ and $\xi$ extend to triple branched coverings $\hat{\eta}: \hat{U} \rightarrow \Delta \cap \mathbb{C}_{\eta\left(J_{\eta}\right)}$ and $\hat{\xi}: \hat{V} \rightarrow \Delta \cap \mathbb{C}_{\xi\left(J_{\xi}\right)}$ respectively. We will turn $\boldsymbol{H}$ into a topological space by identifying it with a subset of $\boldsymbol{B} \times \boldsymbol{B}$ by $\mathcal{H} \mapsto(\hat{U}, 0, \hat{\eta}) \times(\hat{V}, 0, \hat{\xi})$.

As follows immediately from the definition,

Proposition 2.9. The holomorphic commuting pairs based on maps in the standard family belong to $\boldsymbol{H}$.

Let us make a note of an important compactness property of $\mathcal{E}_{s}$

Lemma 2.10. Let $s \in(0,1)$. The collection of normalized maps $g \in \mathcal{E}_{s}$ with $I=[0,1]$, with marked domains $(U, 0)$ is sequentially compact with respect to Carathéodory convergence.

Proof. Let $g_{n}: I=[0,1] \rightarrow J_{n} \subset \tilde{J}_{n}$ be a normalized sequence in $\mathcal{E}_{s}$. These maps extend to triple branched coverings $g_{n}: G_{n} \rightarrow \mathbb{C}_{\tilde{J}_{n}}$; and can be decomposed (2.4) as $g_{n}=Q_{n} \circ h_{n}$ where $Q_{n}(z)=Q_{c_{n}}(z)=z^{3}+c_{n}$, and $h_{n}: I \rightarrow[0, b]$ is a univalent map $G_{n} \rightarrow \Delta\left(h_{n}\right)$. By passing to a subsequence we can ensure that $\tilde{J}_{n}$ converge to an interval $\tilde{J}$, and $c_{n} \rightarrow c$. Then $\left(\Delta\left(h_{n}\right), 0\right)$ Carathédory converges to a slit domain $(\Delta, 0)$.

Since $J_{n}$ is $s^{-1}$-commensurable with $I_{n}, h_{n}(1)$ is bounded. As $g_{n}^{\prime}(1)>s>0$, the derivatives $\left(h_{n}^{-1}\right)^{\prime}\left(h_{n}(1)\right)$ are bounded from above. The points $h_{n}(1)$ stay away from the boundary of $\Delta$, and it follows from Koebe Distortion theorem, that $\left\{h_{n}^{-1}\right\}$ form a normal family in $\Delta$.

Let us select a locally uniformly converging subsequence $h_{n}^{-1}$. Since $h_{n}(I)$ have bounded length, the limit of this sequence is a non-constant, and therefore univalent, function $h^{-1}$ : $\Delta \rightarrow G$. Let $R_{n}: \mathbb{D} \rightarrow G_{n}$ be the normalized Riemann map, $R_{n}(0)=0, R_{n}^{\prime}(0)>0$. It can be decomposed as $R_{n}=h_{n}^{-1} \circ R_{n}^{\prime}$, where $R^{\prime}$ is the normalized Riemann map $\mathbb{D} \rightarrow \Delta\left(h_{n}\right)$. 
By the above, the maps $R_{n}$ converege locally uniformly to the Riemann map $R: \mathbb{D} \rightarrow G$, which is equivalent to Carathéodory convergence $\left(G_{n}, 0\right) \rightarrow(G, 0)$.

Finally, note that the convergence of $h_{n}^{-1}$ implies that there is a point $c \in I$ where derivatives of $h_{n}$ are bounded from above. It follows from Koebe theorem, that $\left\{h_{n}\right\}$ is a normal family in $G$, and hence $h_{n} \rightarrow h \equiv\left(h^{-1}\right)^{-1}$.

The above proof also yields:

Lemma 2.11. For any $s \in(0,1)$, there exists a domain $O_{s} \supset[0,1]$, such that for any $g \in \mathcal{E}_{s}$ with normalization $I=[0,1]$, the univalent map $h$ in (2.4) is well-defined and has $K(s)$-bounded distortion in $O_{s}$.

We will further refer to the above property by saying that a map $g \in \mathcal{E}_{s}$ is cubic up to bounded distortion

The Epstein class of critical circle maps was first introduced by Eckmann and Epstein [EE as an invariant subspace for the Renormalization operator, it was further shown in $[\mathrm{EE}$ that this class contains a fixed point of $\mathcal{R}$ with golden mean rotation number. It can be shown using real distortion estimates, that renormalizations of any smooth circle map converge to the Epstein class, and by recent work of de Faria and de Melo dFdM1, the rate of convergence is geometric in $C^{r}$ metric. We shall only use a the following, weaker statement:

Lemma 2.12. Let $f \in C^{r},(r \geq 3)$ be a critical circle map with an irrational rotation number. Then the collection of real commuting pairs $\left(\widetilde{f^{q_{m+1}}}\left|\widetilde{I_{m}}, \widetilde{f^{q_{m}}}\right| \widetilde{I_{m+1}}\right)$ is precompact in $C^{r}$ topology, and all its partial limits are contained in $\mathcal{E}_{s}$, for some universal constant $s>0$, independent on the original map $f$.

In particular for a critical circle map $f \in \mathcal{E}$ there exists $\sigma>0$ such that all its renormalizations are contained in $\mathcal{E}_{\sigma}$. Moreover, the constant $\sigma$ can be chosen independent on $f$, after skipping first few renormalizations.

Lemma 2.13. Let $\zeta=(\eta, \xi) \in \mathcal{E}$ be a critical commuting pair with $\rho(\zeta)=0$, which appears as a limit of a sequence $\left\{\zeta_{n}\right\} \subset \mathcal{E}$ with $\rho\left(\zeta_{n}\right) \in \mathbb{R} \backslash \mathbb{Q}$. Then the map $\eta$ has a unique fixed point in the interval $I_{\eta}$, which is necessarily parabolic, with multiplier one.

Proof. The existence of a fixed point in $I_{\eta}$ is clear. Since there are arbitrary small real perturbations of $\eta$ without a fixed point on the real line, any such point must be parabolic.

Let us show that the fixed point is unique. We thank P. Jones for suggesting the following argument. Assume that $a$ and $b$ are two distinct fixed points of $\eta$ in $I_{\eta}$. Since $\eta$ is in the Epstein class, it has a well-defined inverse branch $\varphi$ in $\mathbb{C}_{[a, b]}$. As $a$ and $b$ are fixed points, $\varphi([a, b])=[a, b]$. Since $\varphi \neq \mathrm{Id}$, there exists $x_{0} \in[a, b]$ with $\varphi\left(x_{0}\right) \neq x_{0}$. Without loss of generality, assume that $\varphi\left(x_{0}\right)>x_{0}$. Let $\operatorname{dist}_{P}(\cdot, \cdot)$ denote the Poincaré distance in $\mathbb{C}_{[a, b]}$. Set $\epsilon=\operatorname{dist}_{P}\left(\varphi\left(x_{0}\right), x_{0}\right)$. By Schwarz Lemma, $\operatorname{dist}_{P}(\varphi(x), x)>\epsilon$ for all $x<x_{0}$. On the other hand, $\operatorname{dist}_{P}(\varphi(x), x)<$ const $\cdot(x-a)^{-1}$. Therefore, $\varphi^{\prime}(a)=1+\epsilon^{\prime}>1$, a contradiction. 
A commuting pair $\zeta=(\eta, \xi) \in \mathcal{E}$ will be called parabolic if the map $\eta$ has a unique fixed point in $I_{\eta}$, which has a unit multiplier; this point will usually be denoted $p_{\eta}$. Note, that by virtue of its uniqueness, $p_{\eta}$ has to be globally attracting on one side for the interval homeomorphism $\left.\eta\right|_{I_{\eta}}$, it is globally attracting on the other side under $\eta^{-1}$.

Complex bounds. The main analytic result of the paper [Ya1] was establishing complex a priori bounds for Epstein critical circle maps in the following form:

Theorem 2.14 (Ya1]). For any $s \in(0,1)$ there exists $N=N(s)$ and $D=D_{r}(0)$ such that the following holds:

Let $f \in \mathcal{E}_{s}$ be a critical circle map whose rotation number $\rho(f)$ has the continued fraction representation with at least $N+1$ terms. Denote by $\Omega_{m} \ni 0$ the domain which triple covers $\mathbb{C}_{f^{q_{m+1}\left(I_{m}\right)}}$ under $f^{q_{m+1}}$. Then

$$
\frac{\operatorname{dist}\left(f^{q_{m+1}}(z), 0\right)}{\left|f^{q_{m+1}}\left(I_{m}\right)\right|}+d>c\left(\frac{\operatorname{dist}(z, 0)}{\left|I_{m}\right|}\right)^{3} \text {, for all } z \in \Omega_{m} \text {, with } f^{q_{m+1}}(z) \in D \text {. }
$$

The constants $c$ and $d$ in the above inequality is universal.

As an immediate consequence, for $m$ sufficiently large, we can choose a Euclidean disc $\Delta \equiv D_{r_{m}}(0)$ around the origin, commensurable with $I_{m}$, such that the preimages $U_{m}=$ $f^{-q_{m+1}}\left(D_{r_{m}}\right) \cap \Omega_{m}$ and $V_{m}=f^{-q_{m}}\left(D_{r_{m}}\right) \cap \Omega_{m-1}$ are contained in a concentric disc with a smaller radius $r_{m}^{\prime}$, and moreover, $r_{m} / r_{m}^{\prime}$ is greater than some fixed value $K>1$. Thus the real commuting pair $\left(f^{q_{m+1}}\left|I_{m}, f^{q_{m}}\right| I_{m+1}\right)$ extends to a holomorphic pair with range $\Delta$ and definite modulus:

$$
\mathcal{H}:\left(f^{q_{m+1}}: U_{m} \rightarrow \Delta, f^{q_{m}}: V_{m} \rightarrow \Delta\right)
$$

For $\mu \in(0,1)$ let $\boldsymbol{H}(\mu)$ denote the space of holomorphic commuting pairs $\mathcal{H}: \Omega_{<} \rightarrow \Delta_{\mathcal{H}}$, with $\bmod \left(\Delta_{\mathcal{H}} \backslash \Omega_{\zeta}\right)>\mu, \min \left(\left|I_{\eta}\right|,\left|I_{\xi}\right|\right)>\mu$ and $\operatorname{diam}\left(\Delta_{\mathcal{H}}\right)<1 / \mu$.

Lemma 2.15. For each $\mu \in(0,1)$ the space $\boldsymbol{H}(\mu)$ is sequentially pre-compact, with every limit point contained in $\boldsymbol{H}(\mu / 2)$.

Proof. Let $\left\{\mathcal{H}_{n}\right\}$ be a sequence of holomorphic pairs in $\boldsymbol{H}(\mu)$, with ranges $\Delta_{n}$. Let $\hat{\eta}_{n}$ : $\hat{U}_{n} \rightarrow \Delta_{n}$ and $\hat{\xi}_{n}: \hat{V}_{n} \rightarrow \Delta_{n}$ be the three fold extensions of $\eta_{n}, \xi_{n}$.

By passing to a subsequence let us ensure that the Riemann maps $R_{\left(U_{n}, 0\right)}$ converge locally uniformly to a map $R$. It easily follows from Koebe Distortion Theorem that $R$ is non-constant and thus a univalent map $R \equiv R_{(U, 0)}$.

The family $\hat{\eta}_{n}$ is normal in $U$ by Montel's Theorem, and passing to a subsequence again, we have $\left(\hat{U}_{n}, 0, \hat{\eta}_{n}\right) \rightarrow(U, 0, \hat{\eta})$. The convergence of $\hat{\xi}_{n}$ is ensured in the same fashion. Clearly, the resulting pair has modulus greater than $\mu / 2$, and thus is in $\boldsymbol{H}(\mu / 2)$.

Let us define germs of holomorphic commuting pairs using the equivalence relation: $\mathcal{H} \equiv \mathcal{G}$ if $K_{\mathcal{H}}$ and $K_{\mathcal{G}}$ coincide as sets, and $S_{\mathcal{H}} \equiv S_{\mathcal{G}}$ on an open neighborhood of $K_{\mathcal{H}}$. The germ of a pair $\mathcal{H}$ will be denoted $[\mathcal{H}]$. 
Straightening theorems for holomorphic commuting pairs. The existence of extensions to holomorphic pairs with complex a priori bounds for high renormalizations of Epstein circle maps, together with an analysis of the shapes of the domains of these extensions was used in Ya1 to construct quasiconformal conjugacies between high renormalizations of circle maps with the same rotation number, with universally bounded dilatation.

Let $\zeta$ be at least $n$ times renormalizable critical commuting pair. For lack of a better term, let us say that the pair of numbers $\tau_{n}(\zeta)=\left(r_{n-1}, r_{n-2}\right)$ forms the history of the pair $\mathcal{R}^{n} \zeta$

Theorem 2.16 ( Ya1 $)$. For each $s \in(0,1)$ there exists $N=N(s)$, such that the following holds. Let $\zeta_{1}=\left(\eta_{1}, \xi_{1}\right)$ and $\zeta_{2}=\left(\eta_{2}, \xi_{2}\right)$ be two critical commuting pairs in $\mathcal{E}_{s}$ with irrational rotation numbers. Assume that their $N$-th renormalizations have the same rotation number and the same history. Then for every $n \geq N$ the renormalizations $\mathcal{R}^{n} \zeta_{1}$ and $\mathcal{R}^{n} \zeta_{2}$ extend to holomorphic commuting pairs which are $K$-quasiconformally conjugate with a universal dilatation bound $K$.

For commuting pairs of bounded type this Theorem was proved by de Faria dF1, dF2], with " $K$ " depending on the bound on the rotation number.

One of the key points in quadratic-like renormalization theory is Douady-Hubbard Straightening Theorem, which claims that every quadratic-like map is conjugated to a quadratic polynomial via a quasiconformal homeomorphism which is conformal on the Julia set (a so-called hybrid equivalence). In renormalization theory of holomorphic commuting pairs, the role of quadratic polynomials is played by holomorphic pairs generated by standard maps (Lemma 2.4). Below we will establish certain rigidity properties for these pairs, and prove a version of Straightening Theorem for holomorphic commuting pairs.

Lemma 2.17. Let $f_{\theta}$ be a map in the standard family. As before, let $A_{\theta}$ denote its lift to the plane, and $T(z)=z+1$. Let $k \in \mathbb{N} \cup\{\infty\}$ denote the length of the continued fraction expansion of the rotation number $\rho(\theta)$. Suppose that for some $n<k$, the critical commuting pair

$$
\zeta_{\theta, n}=\left(\eta=T^{-p_{n+1}} \circ A_{\theta}^{q_{n+1}}, \xi=T^{-p_{n}} \circ A_{\theta}^{q_{n}}\right)
$$

extends to a holomorphic commuting pair $\mathcal{H}: \Omega \rightarrow \Delta$, where $p_{n} / q_{n}$ is the $n$-th convergent of $\rho(\theta)$. Then any $\mathcal{H}$-invariant Beltrami differential $\mu$ with support in $K(\mathcal{H})$ can be extended off $\Omega$ to a Beltrami differential $\hat{\mu}$ with support in $K\left(A_{\theta}\right)$ which is invariant under $A_{\theta}$ and $T$ (that is $\hat{\mu}$ projects to a Beltrami differential on the quotient cylinder $\mathbb{C} / \mathbb{Z}$, invariant under $\left.f_{\theta}\right)$.

Proof. Set $g_{n}=T^{-p_{n}} \circ A_{\theta}^{q_{n}}: \mathbb{C} \rightarrow \mathbb{C}$. Recall, that if $\rho(\theta)=\left[r_{0}, r_{1}, \ldots\right]$ then $q_{n+1}=$ $r_{n} q_{n}+q_{n-1}$, with $q_{0}=1, q_{1}=r_{0}$, and $p_{n+1}=r_{n} p_{n}+p_{n-1}$, with $p_{0}=0, p_{1}=1$. Let $\mu$ be as above, and consider the Beltrami differential $\mu^{\prime}$ obtained by pulling $\mu$ back by various inverse branches of $g_{n+1}=T^{-p_{n+1}} \circ A_{\theta}^{q_{n+1}}$ and $g_{n}=T^{-p_{n}} \circ A_{\theta}^{q_{n}}$. Observe, that $\mu^{\prime}$ is invariant under $g_{n-1}=g_{n+1} \circ g_{n}^{-r_{n}}$. Arguing inductively, we see that $\mu^{\prime}$ is invariant under $g_{0}=A_{\theta}$ and $g_{1}=T \circ A_{\theta}^{r_{0}}$ and thus under $T$ as well. 
Theorem 2.18 (Straightening). Let $\zeta \in \mathcal{E}$ be a critical commuting pair with an irrational rotation number. Then there exists $N$ such that for all $n>N$ the renormalization $\mathcal{R}^{n}(\zeta)$ is $K$-quasiconformally conjugate to a holomorphic pair $\mathcal{H}$, whose underlying real commuting pair is $\zeta_{\theta, n}$ (2.7) for some $\theta \in[0,1), n \in \mathbb{N}$. The constant $K$ is universal, and the conjugacy is conformal on the filled Julia set $K(\mathcal{H})$.

Proof. The existence of the conjugacy is guaranteed by Theorem 2.16. Its conformality on the filled Julia set follows from Lemmas 2.17 and 2.7.

Theorem 2.19 (Straightening of limiting pairs). Let $\zeta \in \mathcal{E}$ be a critical commuting pair with an irrational rotation number. Assume that there is a sequence $\left\{n_{k}\right\} \subset \mathbb{N}$, such that some holomorphic pair extensions $\mathcal{H}_{k}$ of $\mathcal{R}^{n_{k}} \zeta$ converge in $\boldsymbol{H}$ to a pair $\mathcal{H}: \Omega_{\mathcal{H}} \rightarrow \Delta_{\mathcal{H}}$, which is parabolic. Assume in addition, that $\tau_{n_{k}}(\zeta)=(a, b)$.

Then we can find $\theta=\theta(a, b) \in[0,1)$ such that the critical commuting pair $\zeta_{\theta, N}$ extends to a parabolic holomorphic commuting pair $\mathcal{G}: \Omega_{\mathcal{G}} \rightarrow \Delta_{\mathcal{G}}$ with history $(a, b)$; there exists a $K$-quasiconformal map $\phi: \Delta_{\mathcal{H}} \rightarrow \Delta_{\mathcal{G}}$ which is a conjugacy: $\mathcal{H}=\phi^{-1} \circ \mathcal{G} \circ \phi$. The constant $K$ is universal, and $\phi$ is conformal on the filled Julia set $K(\mathcal{H})$.

Proof. The existence of $\mathcal{G}, K$-quasiconformally conjugate to $\mathcal{H}$ is guaranteed by Theorem 2.16 and compactness of quasiconformal maps. Let $\psi: \Delta_{\mathcal{H}} \rightarrow \Delta_{\mathcal{G}}$ be a conjugacy. Consider a new complex structure $\mu$ on $\Delta_{\mathcal{G}}$ given by the pullback $\left(\phi^{-1}\right)^{*} \sigma_{0}$ on $K(\mathcal{G})$ and the standard structure $\sigma_{0}$ elsewhere.

By Lemma 2.17 the structure $\mu$ can be extended to a global structure $\mu^{\prime}$ invariant under $A_{\theta}$ and $T$. Let $q: \mathbb{C} \rightarrow \mathbb{C}$ be the solution of $\bar{\partial} q / \partial q=\mu^{\prime}$ fixing 0,1 , and $\infty$. By Lemma 2.6, $q \circ \mathcal{G} \circ q^{-1}=\mathcal{G}$. The map $\phi=q \circ \psi$ is the conjugacy with the desired properties.

\section{PARABOlic points, their PERTURbations, AND PARABOliC RENORMALizATION}

General theory. We begin with a brief review of the theory of parabolic bifurcations, as applied in particular to an interval map in the Epstein class. For a more comprehensive exposition the reader is referred to [Do], supporting technical details may be found in [Sh]. Fix a map $\eta_{0} \in \mathcal{E}$ having a parabolic fixed point $p$ with unit multiplier.

Theorem 3.1 (Fatou Coordinates). There exist topological discs $U^{A}$ and $U^{R}$, called attracting and repelling petals, whose union is a punctured neighborhood of the parabolic periodic point $p$ such that

$$
\begin{gathered}
\eta_{0}\left(\bar{U}^{A}\right) \subset U^{A} \bigcup\{p\}, \text { and } \bigcap_{k=0}^{\infty} \eta_{0}^{k}\left(U^{A}\right)=\{p\}, \\
\eta_{0}\left(\bar{U}^{R}\right) \subset U^{R} \bigcup\{p\}, \text { and } \bigcap_{k=0}^{\infty} \eta_{0}^{-k}\left(U^{R}\right)=\{p\},
\end{gathered}
$$

where $\eta_{0}^{-1}$ is the univalent branch fixing $\zeta$. 
Moreover, there exist injective analytic maps

$$
\Phi^{A}: U^{A} \rightarrow \mathbb{C} \text { and } \Phi^{R}: U^{R} \rightarrow \mathbb{C},
$$

unique up to post-composition by translations, such that

$$
\Phi^{A}\left(\eta_{0}(z)\right)=\Phi^{A}(z)+1 \text { and } \Phi^{R}\left(\eta_{0}(z)\right)=\Phi^{R}(z)+1 .
$$

The Riemann surfaces $C^{A}=U^{A} / \eta_{0}$ and $C^{R}=U^{R} / \eta_{0}$ are conformally equivalent to the cylinder $\mathbb{C} / \mathbb{Z}$.

We denote $\pi_{A}: U^{A} \rightarrow C^{A}$ and $\pi_{R}: U^{R} \rightarrow C^{R}$ the natural projections. The quotients $C^{A}$ and $C^{R}$ are customarily referred to as Ecalle-Voronin cylinders; we will find it useful to regard these as Riemann spheres with distinguished points \pm filling in the punctures. The real axis projects to natural equators $E^{A} \subset C^{A}$ and $E^{R} \subset C^{R}$. Any conformal transit homeomorphism $\tau: C^{A} \rightarrow C^{R}$ fixing the ends \pm is a translation in suitable coordinates. Lifiting it produces a map $\bar{\tau}: U^{A} \rightarrow \mathbb{C}$ satisfying

$$
\tau \circ \pi_{A}=\pi_{R} \circ \bar{\tau} .
$$

We will sometimes write $\tau \equiv \tau_{\theta}$, and $\bar{\tau}=\bar{\tau}_{\theta}$, where

$$
\Phi^{R} \circ \bar{\tau} \circ\left(\Phi^{A}\right)^{-1}(z) \equiv z+\theta \bmod (z) .
$$

If $z \in U^{A} \cap U^{R}$, we set $\mathrm{E}\left(\pi_{R}(z)\right)=\pi_{A}(z)$. This Écalle-Voronin transformation extends to an analytic map of the neighborhoods $W(+), W(-)$ of the two ends of $C_{R}$. Composing with a transit homeomorphism yields an analytic dynamical system $F_{\tau}=\tau \circ \mathrm{E}: W(+) \cup W(-) \rightarrow$ $C^{R}$ with fixed points \pm . The product of corresponding eigenvalues is clearly independent of $\tau$; noting that each of the components $W( \pm)$ is mapped onto the whole $C^{R}$ and applying Schwarz Lemma we conclude that

$$
\left|F_{\tau}^{\prime}(+)\right| \cdot\left|F_{\tau}^{\prime}(-)\right|>1 .
$$

For an Epstein map $\eta$ in a sufficiently small neighborhood of $\eta_{0}$ the parabolic point splits into a complex conjugate pair of repelling fixed points $p_{\eta} \in \mathbb{H}$ and $\bar{p}_{\eta}$ with multipliers $e^{2 \pi i \pm \alpha(\eta)}$. In this situation one may still speak of attracting and repelling petals:

Lemma 3.2 (Douady Coordinates). There exists a neighborhood $U\left(\eta_{0}\right) \subset \mathcal{E}$ of the map $\eta_{0}$ such that for any $\eta \in U\left(\eta_{0}\right)$ with $|\arg \alpha(\eta)|<\pi / 4$, there exist topological discs $U_{\eta}^{A}$ and $U_{\eta}^{R}$ whose union is a neighborhood of $p$, and injective analytic maps

$$
\Phi_{\eta}^{A}: U^{A} \rightarrow \mathbb{C} \text { and } \Phi_{\eta}^{R}: U_{f}^{R} \rightarrow \mathbb{C}
$$

unique up to post-composition by translations, such that

$$
\Phi_{\eta}^{A}(\eta(z))=\Phi_{\eta}^{A}(z)+1 \text { and } \Phi_{\eta}^{R}(\eta(z))=\Phi_{\eta}^{R}(z)+1 .
$$

The quotients $C_{\eta}^{A}=U_{\eta}^{A} / \eta$ and $C_{\eta}^{R}=U_{\eta}^{R} / \eta$ are Riemann surfaces conformally equivalent to $\mathbb{C} / \mathbb{Z}$. 
Note that the condition on arguments of the eigenvalues of the repelling fixed points of $\eta$ is automatically satisfied for all maps in a sufficiently small neighborhood of $\eta_{0}$ as follows from Holomorphic Index formula.

An arbitrary choice of real basepoints $a \in U^{A}$ and $r \in U^{R}$ enables us to specify the Fatou and Douady coordinates uniquely, by requiring that $\Phi^{A}(a)=\Phi_{\eta}^{A}(a)=0$, and $\Phi^{R}(r)=\Phi_{\eta}^{R}(r)=0$. The following fundamental theorem first appeared in [DH]:

Theorem 3.3. With these normalizations we have

$$
\Phi_{\eta}^{A} \rightarrow \Phi^{A} \text { and } \Phi_{\eta}^{R} \rightarrow \Phi^{R}
$$

uniformly on compact subsets of $U^{A}$ and $U^{R}$ respectively.

Moreover, select the smallest $n(\eta) \in \mathbb{N}$ for which $\eta^{n(\eta)}(a) \geq r$. Then

$$
\eta^{n(\eta)}(z)=\left(\Phi_{\eta}^{R}\right)^{-1} \circ T_{\theta(\eta)+K} \circ \Phi_{\eta}^{A}
$$

wherever both sides are defined. In this formula $T_{a}(z)$ denotes the translation $z \mapsto z+a$, $\theta(\eta) \in[0,1)$ is given by

$$
\theta(\eta)=1 / \alpha(\eta)+\underset{\alpha(\eta) \rightarrow \infty}{o(1)} \bmod 1,
$$

and the real constant $K$ is determined by the choice of the basepoints a, $r$. Thus for a sequence $\left\{\eta_{k}\right\} \subset U(\eta)$ converging to $\eta$, the iterates $\eta_{k}^{n\left(\eta_{k}\right)}$ converge locally uniformly if and only if there is a convergence $\theta(\eta) \rightarrow \theta$, and the limit in this case is a certain lift of the transit homeomorphism $\tau_{\theta}$ for the parabolic map $\eta_{0}$.

Parabolic renormalization of commuting pairs. Let $\zeta=(\eta, \xi) \in \mathcal{E}$ be a parabolic commuting pair. Denote by $p \in I_{\eta}$ the parabolic fixed point of $\eta$. The pair $\zeta$ has zero rotation number and is, therefore, not renormalizable. In what follows we will use the above discussed local theory of parabolic germs to describe a parabolic renormalization construction for a parabolic pair $\zeta$, which will naturally supplement the usual renormalization procedure. A parallel construction for parabolic quadratic-like maps appeared in a paper of Douady and Devaney [DD].

Let $U^{A}, U^{R}, C^{A}$, and $C^{R}$ be as above. Fix an arbitrary transfer isomorphism $\tau$ : $C^{A} \rightarrow C^{R}$ which preserves the equators (i.e. a rigid rotation). Let $N \geq 0$ be such that $\eta^{N}(\xi(0)) \in C^{A}$. Fix a branch $\pi_{R}^{-1}$ and take the smallest $M$ for which

$$
\eta^{M} \circ \pi_{R}^{-1} \circ \tau \circ \pi_{A} \circ \eta^{N} \circ \xi(0) \in[0, \eta(0)] .
$$

Clearly, the composition

$$
\gamma \equiv \eta^{M} \circ \pi_{R}^{-1} \circ \tau \circ \pi_{A} \circ \eta^{N} \circ \xi
$$

has a well-defined extension to the whole interval $[0, \eta(0)]$ which is independent of the choice of the branch of $\pi_{R}^{-1}$.

Definition 3.1. The parabolic renormalization of the commuting pair $\zeta=(\eta, \xi)$, corresponding to the transit map $\tau$, is the normalized pair

$$
\left.\mathcal{P}_{\tau} \zeta=(\gamma \mid \widetilde{[0, \eta(0)}], \eta \mid \widetilde{[\gamma(0), 0]}\right) \text {. }
$$


Again, we will refer to the non-rescaled commuting pair $(\gamma, \eta)$ as the pre-parabolic renormalization $p \mathcal{P}_{\tau} \zeta$ of the pair $\zeta$.

As an immediate consequence of continuity of Douady coordinates (Theorem 3.3) we have the following:

Lemma 3.4. Let $\zeta_{k}=\left(\eta_{k}, \xi_{k}\right) \in \mathcal{E}$ be a converging sequence of renormalizable commuting pairs, $\zeta_{k} \rightarrow \zeta=(\eta, \xi)$, with rotation numbers $\rho_{k} \rightarrow 0$. Assume that their renormalizations also converge, $\mathcal{R} \zeta_{k} \rightarrow \tilde{\zeta}$. Then for some choice of transit map $\tau$ for the map $\eta$, we have

$$
\mathcal{P}_{\tau} \zeta=\tilde{\zeta}
$$

In the same way as for the renormalization operator $\mathcal{R}$ (see Remark 2.1) we have

Remark 3.1. The parabolic renormalization is injective. More precisely, for any $\zeta \in \boldsymbol{C}$ there exists at most one parabolic commuting pair $\zeta_{-1} \in \boldsymbol{C}_{\infty}$ and a unique choice of the transit map $\tau$ such that $\mathcal{P}_{\tau} \zeta_{-1}=\zeta$.

Proof. Let $\zeta=(\eta, \xi) \in \boldsymbol{C}_{\infty}$ be a parabolic pair, and let $\hat{\zeta}=(\gamma, \eta)=p \mathcal{P}_{\tau} \zeta$. We can write $\gamma=\eta^{M} \circ \bar{\tau} \circ \eta^{N} \circ \xi$ for a choice of local lift $\bar{\tau}$ of the transit map $\tau$ (note that all the maps in this composition commute). The number $N$ can be identified as follows. Let $\bar{\tau}_{1}$ be a lift of an arbitrary transit map, then $N$ is the smallest natural number for which

$\eta^{-(N+M)} \circ \bar{\tau}_{1}^{-1} \circ \gamma$ has a smooth inverse near $\eta(0)$. The position of $\eta^{M} \circ \bar{\tau} \circ \eta^{N} \circ \xi(0)$ depends on $\tau$ monotonously, which, in turn, specifies $\tau$.

\section{TOWERS OF HOLOMORPHIC COMMUTING PAIRS}

Definition 4.1. A tower of Epstein holomorphic commuting pairs is an element of a product space $\boldsymbol{H}^{N}, N \leq \infty$,

$$
\mathcal{T}=\left(\mathcal{H}_{i}\right)_{i=1}^{N}
$$

for which the following holds. If $\zeta_{i}=\left(\eta_{i}, \xi_{i}\right)$ denotes the real commuting pair underlying $\mathcal{H}_{i}$, then either $\chi\left(\zeta_{i}\right) \neq \infty$, and $\tilde{\zeta}_{i+1}=p \mathcal{R} \zeta_{i}$, or $\chi\left(\zeta_{i}\right)=\infty$, and $\tilde{\zeta}_{i+1}=p \mathcal{P}_{\tau_{i}} \zeta_{i}$, for some choice of the transit isomorphism $\tau_{i}$, for all $i \leq N$.

The pair $\mathcal{H}_{1}$ will be referred to as the base pair of the tower $\mathcal{T}$. We shall denote by $\boldsymbol{T}$ the space of all towers. For an element $\mathcal{T} \in \boldsymbol{T}$ its rotation number $\rho(\mathcal{T})$ is the sequence

$$
\rho(\mathcal{T})=\left(\chi\left(\zeta_{1}\right), \chi\left(\zeta_{2}\right), \ldots\right)
$$

The domain and range of the tower $\mathcal{T}$ is the domain $\Omega_{1}$ and range $\Delta_{1}$ of the base pair $\mathcal{H}_{1}$.

The dynamics $F(\mathcal{T})$ associated to the tower $\mathcal{T}$ is the collection of all finite compositions $f=h_{1} \circ \cdots \circ h_{k}$, where $h_{n}$ is an element of $\mathcal{H}_{i}$ or a local lift of some $\tau_{i}$. For a point $z \in \Omega_{1}$, the orbit of $z$ under $\mathcal{T}$ is the set $\mathcal{O}_{\mathcal{T}}(z)$ of images of $z$ under all elements of $F(\mathcal{T})$ which are defined at $z$. We say that $z$ is an escaping point if $\mathcal{O}_{\mathcal{T}}(z) \cap \Delta_{1} \backslash \Omega_{1} \neq \emptyset$. Non-escaping points form the filled Julia set $K(\mathcal{T})$, its boundary is the Julia set $J(\mathcal{T})$. 
Two towers $\mathcal{T}_{1}=\left(\mathcal{H}_{i}^{1}\right)_{i=1}^{N}$ and $\mathcal{T}_{2}=\left(\mathcal{H}_{i}^{2}\right)_{i=1}^{N}$ with domains $\Omega_{1}$ and $\Omega_{2}$ are conjugate (quasiconformally, conformally, etc.) if there is a homeomorphism $\phi$ defined on the neighborhood of the filled Julia set of $\mathcal{T}_{1}$ having the appropriate smoothness, satisying

$$
\mathcal{H}_{i}^{2} \circ \phi=\phi \circ \mathcal{H}_{i}^{1} \text { for all } i \leq N .
$$

We will mostly be concerned with infinite towers (that is $N=\infty$ ).

Examples of towers are readily provided by holomorphic pairs with irrational rotation numbers. More generally, let $\zeta_{i}=\left(\eta_{i}, \xi_{i}\right)$ be a sequence of Epstein commuting pairs with irrational rotation numbers, with holomorphic extensions $\mathcal{H}_{i} \in \boldsymbol{H}$. Suppose that the $n$-th pre-renormalizations $p \mathcal{R}^{n} \zeta_{i}$ have holomorhic pair extensions $\mathcal{H}_{i}^{n}$ which converge for every $n \leq N$ to some $\hat{\mathcal{H}}^{i}$. As follows from Lemma 3.4 , the sequence $\hat{\mathcal{T}}=\left(\hat{\mathcal{H}}_{1}, \hat{\mathcal{H}}_{2}, \ldots, \hat{\mathcal{H}}, \ldots\right)$ forms a tower. Such towers will be referred to as limiting. Theorem 3.3 implies the following:

Proposition 4.1. Let $\hat{\mathcal{T}}$ be a limiting tower as above. For any map $f \in F(\mathcal{T})$, and an open subset $W$ compactly contained in the domain of definition of $f$, there exists a sequence $\left\{f_{k}\right\}$ of finite compositions of elements of $\mathcal{H}_{i}^{n}$ such that $f_{k} \rightrightarrows f$ in the domain $W$.

We will distinguish the case when $\zeta_{i}$ are pre-renormalizations of the same pair, $\zeta_{i}=$ $p \mathcal{R}^{n_{i}} \zeta$, by referring to $\hat{\mathcal{T}}$ as a renormalization limiting tower.

Finally, we will say that a limiting tower $\hat{\mathcal{T}}$ is a standard tower if its base pair $\mathcal{H}_{1}$ is a limit of pairs $\mathcal{G}_{i}$ with $\left.\mathcal{G}_{i}\right|_{\mathbb{R}}=\zeta_{\theta_{i}, n_{i}}$.

The main objective of this section is proving the following Combinatorial Rigidity Theorem for renormalization limiting towers:

Theorem 4.2. Any two renormalization limiting towers with the same rotation number are conjugate by a quasiconformal homeomorphism, which is conformal on the filled Julia sets of the towers.

The Theorem will follow from a rigidity statement for standard towers, to which it is reduced with the help of the Straightening Theorems.

Combinatorial rigidity of standard towers. We begin the proof of Theorem 4.2 by establishing the following fact about standard towers:

Theorem 4.3. Let $\mathcal{T}=\left(\mathcal{H}_{i}\right)$ and $\tilde{\mathcal{T}}=\left(\tilde{\mathcal{H}}_{i}\right)$ be two standard towers. Assume that the base real commuting pairs $\zeta_{1}=\left.\mathcal{H}_{1}\right|_{\mathbb{R}}$ and $\tilde{\zeta}_{1}=\left.\tilde{\mathcal{H}}_{1}\right|_{\mathbb{R}}$ and the rotation numbers $\rho(\mathcal{T})$ and $\rho(\tilde{\mathcal{T}})$ coincide.

Then $\left.\mathcal{H}_{i}\right|_{\mathbb{R}}=\left.\tilde{\mathcal{H}}_{i}\right|_{\mathbb{R}}$ for all $i \in \mathbb{N}$.

The claim of the Theorem 4.3 will evidently follow from

Lemma 4.4. Let $\zeta \in \mathcal{E}$ be a parabolic commuting pair. Let $\left\{r_{i}\right\}_{1}^{m}, m \leq \infty$ be a sequence of positive integers, ending in $\infty$ if $m$ is finite, and set $\rho=\left[r_{1}, \ldots\right]$.

There exists a unique choice of the transit map $\tau$ for which $\rho\left(\mathcal{P}_{\tau} \zeta\right)=\left[r_{1}, \ldots\right]$, and in the case when $\rho \in \mathbb{Q}$ (that is $m<\infty) \mathcal{R}^{m-1}\left(\mathcal{P}_{\tau} \zeta\right)$ is a parabolic pair. 
Proof. We need to introduce some further notation. Let $p$ denote the parabolic fixed point of $\eta$, and let $C^{A}$, and $C^{R}$ be as in $\oint$. The dynamics of the commuting pair $(\eta, \xi)$ induces a natural return map of the equators of the Écalle cylinders $r: E^{R} \rightarrow E^{A}$. For a choice of the transit isomorphism $\tau: C^{A} \rightarrow C^{R}$ fixing the equators the composition

$$
f_{\tau}=\tau \circ r: E^{A} \rightarrow E^{A}
$$

is a critical circle map. It is easily seen that $f_{\tau}$ is topologically conjugate to the circle map associated to $\mathcal{P}_{\tau} \zeta$ (see Proposition 2.1), thus

$$
\rho\left(f_{\tau}\right)=\rho\left(\mathcal{P}_{\tau} \zeta\right)
$$

Note that the dependence $\tau \mapsto f_{\tau}(x)$ is a monotone map $\mathbf{T} \rightarrow \mathbf{T}$ for any $x \in T$. The standard considerations then imply that $\psi: \tau \mapsto \rho\left(f_{\tau}\right)$ is a non-decreasing degree one continuous map of the circle; moreover $\psi^{-1}(\rho)$ is a single point for each irrational $\rho$.

It remains therefore to consider the case when $\rho=p / q$ is rational. The existence of a $\tau$ satisfying the conditions of the Lemma follows from Lemma 3.4, let us establish its uniqueness. Let $F_{\tau}: \mathbb{R} \rightarrow \mathbb{R}$ be a lift of $f_{\tau}$ having singularities at integer points, with $F(0) \in[0,1)$. The map $f_{\tau}$ has rotation number $p / q$ if and only if $F_{\tau}^{q}(x)=x+p$ for some $x \in \mathbb{R}$. If $\tau$ satisfies the conditions of the Lemma, the pair $\mathcal{P}_{\tau} \zeta$ has a unique periodic orbit. The uniqueness of the orbit implies that $F(x) \leq x$ for all $x$, or $F(x) \geq x$ for all $x$. Monotone dependence of $f_{\tau}$ on $\tau$ now implies that the value of $\tau$ realizing each of these two possibilities is unique. Moreover, since the family $f_{\tau}$ contains no rigid rotations, the two values are distinct. Finally we note that by our conventions the continued fraction expansions of $\rho\left(\mathcal{P}_{\tau} \zeta\right)$ in these two situations will differ.

Theorems 2.6 and 4.3 have the following important consequence:

Theorem 4.5. A standard tower admits no non-trivial quasiconformal deformations entirely supported on the filled Julia set of the base pair.

Proof of Theorem 4.2. Let $\mathcal{T}=\left(\mathcal{H}_{1}, \mathcal{H}_{2}, \ldots\right)$ be a limiting tower for a sequence $\mathcal{T}_{i}$ of towers based on the extensions $\mathcal{Z}_{i}$ of the renormalizations $\mathcal{R}^{k_{i}} \zeta$ of a commuting pair $\zeta \in \mathcal{E}$. By Theorem 2.18, there exists a sequence of standard pairs $\mathcal{G}_{i}$ quasiconformally conjugate to $\mathcal{Z}_{i}$ via a homeomorphism $\phi_{i}$ with a uniform dilatation bound. We can select the sequence $\mathcal{G}_{i}$ in such a way that their underlying pairs $\zeta_{\theta_{i}, n_{i}}$ converge to a pair $\hat{\zeta}$. There is no canonical choice for $\hat{\zeta}$, but we may associate a fixed $\hat{\zeta}$ to all renormalization limiting towers $\mathcal{T}$ with the same $\rho(\mathcal{T})$. Using Theorem 2.14, Lemma 2.15 and compactness properties of quasiconformal maps, we may select a subsequence $\phi_{k_{i}}$ converging to a quasiconformal homeomorphism $\phi$, conjugating $\mathcal{H}_{1}: \Omega_{1} \rightarrow \Delta_{1}$ to a pair $\mathcal{G}$ with $\left.\mathcal{G}\right|_{\mathbb{R}}=\hat{\zeta}$ :

$$
\mathcal{H}_{1} \circ \phi=\phi \circ \mathcal{G} \text {. }
$$

Let us denote by $\mathcal{S}$ the standard tower with base $\mathcal{G}$, it is unique by Theorem 4.3 .

We define a new Beltrami differential $\mu$ on $\Delta_{1}$ by first setting $\mu=\phi^{*} \sigma_{0}$ for $z \in \Delta_{1} \backslash \Omega_{1}$. For a point $z$ in $\Omega_{1}$ whose orbit enters $\Delta_{1} \backslash \Omega_{1}$ we set $\mu=g^{*} \phi^{*} \sigma_{0}$, where $g$ is a composition 
of the elements of $\mathcal{T}$ mapping $z$ to $\Delta_{1} \backslash \Omega_{1}$. On the filled Julia set $K(\mathcal{T})$ we set $\mu=\sigma_{0}$. Defined in this way, $\mu$ is invariant under the tower $\mathcal{T}$. Let $h: \Delta_{1} \rightarrow \mathbb{C}$ be the quasiconformal homeomorphism integrating $\mu, \mu=h^{*} \sigma_{0}$. Then $S^{\prime} \equiv h \circ \mathcal{T} \circ h^{-1}$ is a tower. The base pair of $S^{\prime}$ is a quasiconformal deformation $h \circ \phi^{-1} \circ \mathcal{G} \circ \phi \circ h^{-1}$ of $\mathcal{G}$ entirely supported on its filled Julia set. It is thus equal to $\mathcal{G}$ on a neighborhood of the filled Julia set. The rotation number of the standard tower $S^{\prime}$ is equal to $\rho(\mathcal{T})$ and therefore, by Theorem $4.2, S^{\prime}=S$. Q.e.d.

4.1. Bi-infinite towers. A bi-infinite tower is an element of the product space $\boldsymbol{H}^{\mathbb{Z}}$

$$
\mathcal{T}=\left(\ldots, \mathcal{H}_{-n}, \ldots \mathcal{H}_{0}, \ldots, \mathcal{H}_{n}, \ldots\right)
$$

with the following properties. For each $n \in \mathbb{Z}$ setting $\zeta_{i}=\left(\eta_{i}, \xi_{i}\right)=\mathcal{H}_{i} \cap \mathbb{R}$ we either have $\chi\left(\zeta_{i}\right) \neq \infty$ and $\tilde{\zeta}_{i+1}=p \mathcal{R} \zeta_{i}$; or $\chi\left(\zeta_{i}\right)=\infty$ and $\tilde{\zeta}_{i+1}=p \mathcal{P}_{\tau_{i}} \zeta_{i}$ for some choice of the transit map $\tau_{i}$. The rotation number of $\mathcal{T}$ is naturally defined to be the bi-infinite sequence

$$
\rho(\mathcal{T})=\left(\ldots, \chi\left(\zeta_{-i}\right), \ldots, \chi\left(\zeta_{0}\right), \ldots, \chi\left(\zeta_{i}\right), \ldots\right) .
$$

Examples of bi-infinte towers can be constructed from renormalizations of Epstein commuting pairs with irrational rotation numbers as follows. Let $\zeta_{k} \in \mathcal{E}$ with $\rho\left(\zeta_{k}\right) \in \mathbb{R} \backslash \mathbb{Q}$. Let

$$
\mathcal{T}_{k}=\left(\mathcal{H}_{-i_{k}}^{k}, \mathcal{H}_{-i_{k}+1}^{k}, \ldots, \mathcal{H}_{0}^{k}, \mathcal{H}_{1}^{k}, \ldots\right)
$$

be a forward tower of holomorphic pair extensions of the renormalizations of $\zeta_{k}$

$$
\mathcal{H}_{j-i_{k}}^{k} \cap \mathbb{R}=\lambda_{k}^{-1} \circ \mathcal{R}^{j} \zeta_{k} \circ \lambda_{k}
$$

where the homothety $\lambda_{k}$ is chosen so that $\tilde{\mathcal{H}}_{0}^{k}=\mathcal{H}_{0}^{k}$. If $\zeta_{k}$ are selected so that $i_{k} \rightarrow \infty$ and also $\mathcal{H}_{n}^{k} \underset{k \rightarrow \infty}{\longrightarrow} \mathcal{H}^{k}$ for all $n \in \mathbb{Z}$ then the sequence $\mathcal{T}=\left(\mathcal{H}^{k}\right)_{-\infty}^{\infty}$ is a bi-infinite tower. If in addition $\zeta_{k}=\mathcal{R}^{n_{k}} \zeta$ for some $\zeta \in \mathcal{E}$ then $\mathcal{T}$ is a limiting renormalization tower.

The main result of this paper transforms into the following uniqueness theorem for biinfinite limiting renormalization towers:

Theorem 4.6. Let $\mathcal{T}^{1}=\left(\mathcal{H}_{k}^{1}\right)_{-\infty}^{\infty}$ and $\mathcal{T}^{2}=\left(\mathcal{H}_{k}^{2}\right)_{-\infty}^{\infty}$ be two limiting renormalization towers with the same rotation number. Then

$$
\mathcal{H}_{k}^{1} \cap \mathbb{R}=\lambda^{-1} \circ \mathcal{H}_{k}^{2} \circ \lambda
$$

for some real homothety $\lambda$ for all $k \in \mathbb{Z}$.

The proof of Theorem 4.6 is broken into two steps. We first establish

Theorem 4.7. Let $\mathcal{T}^{1}=\left(\mathcal{H}_{k}^{1}\right)_{-\infty}^{\infty}$ and $\mathcal{T}^{2}=\left(\mathcal{H}_{k}^{2}\right)_{-\infty}^{\infty}$ be two limiting renormalization towers with the same rotation number. Then there exists a quasiconformal homeomorphism $\phi: \mathbb{C} \rightarrow \mathbb{C}$ conjugating the towers:

$$
\mathcal{H}_{k}^{1}=\phi^{-1} \circ \mathcal{H}_{k}^{2} \circ \phi, \text { for all } k \in \mathbb{Z} .
$$


Proof. By Theorem 4.2 the towers

$$
\mathcal{T}_{n}^{1}=\left(\mathcal{H}_{n}^{1}, \mathcal{H}_{n+1}^{1}, \ldots\right) \text { and } \mathcal{T}_{n}^{2}=\left(\mathcal{H}_{n}^{2}, \mathcal{H}_{n+1}^{2}, \ldots\right)
$$

are quasiconformally conjugate by a homeomorphism $\phi_{n}: \Delta_{n}^{1} \rightarrow \Delta_{n}^{2}$ of the domains of $\mathcal{H}_{n}^{1}$ and $\mathcal{H}_{n}^{2}$ respectively, whose dilatation is bounded by a universal constant. By complex bounds, the domains $\Delta_{n}^{i}$ fill out the plane; choosing a diagonal subsequence of maps $\phi_{n_{i}}$ converging in each $\Delta_{n}$ we obtain a limiting quasiconformal mapping $\phi: \mathbb{C} \rightarrow \mathbb{C}$ with the required properties.

We now proceed to formulate

Theorem 4.8. A limiting renormalization bi-infinite tower admits no nontrivial invariant Beltrami differentials.

Theorem 4.8 readily implies Theorem 4.6, since the Beltrami differential of the quasiconformal conjugacy $\phi$ produced by Theorem 4.7 is invariant under the tower $\mathcal{T}^{1}$, and so equal to zero almost everywhere. Thus the conjugacy $\phi: \mathbb{C} \rightarrow \mathbb{C}$ is conformal and therefore a homothety. The proof of Theorem 4.8 is based on hyperbolic metric expansion techniques developed by McMullen in [McM2] and used by Hinkle in the context parallel to ours in [Hin]. It will occupy most of the remainder of the paper.

4.2. Expansion of the hyperbolic metric. We fix a bi-infinite renormalization limiting tower $\mathcal{T}=\left(\mathcal{H}_{k}\right)_{-\infty}^{\infty}, \mathcal{H}_{k} \cap \mathbb{R}=\zeta_{k}=\left(\eta_{k}, \xi_{k}\right)$. We will refer to $h \in F(\mathcal{T})$ as a map of level $k$ if $h$ is either an element of the holomorphic commuting pair $\mathcal{H}_{k}$, that is one of the maps $\eta_{k}, \xi_{k}$, or $\eta_{k} \circ \xi_{k}$, or a lift of a transit homeomorphism $\tau$ associated to the parabolic point of $\eta_{k}$. The domain of $h$, denoted by $D(h)$, will in the first case denote the domain of the extension of $h$ to a degree three proper map onto the plane; and in the case when $h$ is a lift of a transit map it will be the domain of the maximal extension of $h$ provided by the Fatou coordinates.

Given a hyperbolic Riemann surface $X$ we shall denote by $d_{X}(\cdot, \cdot)$ the Poincaré distance on $X$. For a differentiable map $f: X \rightarrow Y$ of hyperbolic Riemmann surfaces, $\left\|f^{\prime}(x)\right\|_{X, Y}$ will stand for the norm of the derivative with respect to the hyperbolic metrics; we will simply write $\left\|f^{\prime}(x)\right\|$ if $X=Y=\mathbb{C} \backslash \mathbb{R}$. The expansion properties of bi-infinite towers rely on the following lemma formulated by McMullen in McM2]:

Lemma 4.9. There exists a continuous and increasing function $C(s)<1$ with $C(s) \rightarrow 0$ as $s \rightarrow 0$ such that for the inclusion $\iota$ of a hyperbolic Riemann surface $X$ into a hyperbolic Riemann surface $Y$,

$$
\left\|\iota^{\prime}(z)\right\|_{X, Y}<C(s)
$$

where $s=d_{Y}(x, Y \backslash X)$.

The following estimate for the variation of the norm of the derivative of an analytic map of hyperbolic Riemann surfaces is a consequence of Koebe Distortion Theorem: 
Lemma 4.10 (Corollary 2.27 [McM1]). Let $f: X \rightarrow Y$ be an analytic map between hyperbolic Riemann surfaces with nowhere vanishing derivative. Then for $z_{1}, z_{2} \in X$ we have

$$
\left\|f^{\prime}\left(z_{1}\right)\right\|_{X, Y}^{1 / \alpha} \leq\left\|f^{\prime}\left(z_{2}\right)\right\|\left\|_{X, Y} \leq\right\| f^{\prime}\left(z_{1}\right) \|_{X, Y}^{\alpha},
$$

where $\alpha=\exp \left(K d_{X}\left(z_{1}, z_{2}\right)\right)$ with a universal constant $K>0$.

We now apply the above expansion principles to the setting of limiting renormalization towers:

Lemma 4.11. Let $\mathcal{T}=\left(\mathcal{H}_{1}, \mathcal{H}_{2}, \ldots\right)$ be a limiting renormalization tower, $h \in F(\mathcal{T})$. As before, denote by $\Omega_{n}=U_{n} \cup V_{n} \cup D_{n}$ and $\Delta_{n}$ the domain and range of the holomorphic pair $\mathcal{H}_{n}$. We have the following:

(I) $\left\|h^{\prime}(z)\right\|>1$ for any $x \in D(h)$;

(II) There exists a universal constant $C>1$ such that $\left\|h^{\prime}(z)\right\|>C$ if $z \in \Omega_{n}$ and $h$ is an element of level $n$ for which $h(x) \in \Delta_{n} \backslash \Omega_{n}$.

Proof. Let us consider a holomorphic commuting pair $\mathcal{H}$ which is an extension of a high renormalization $\mathcal{R}^{n} \zeta$ for $\zeta \in \mathcal{E}$ with $\rho(\zeta) \in \mathbb{R} \backslash \mathbb{Q}$. Following the earlier convention, we write

$$
\mathcal{H}=\left(\eta=\left.f^{q_{n+1}}\right|_{U}, \xi=\left.f^{q_{n}}\right|_{V}\right) .
$$

The pair $\mathcal{H}$ should be viewed as a small perturbation of an element of $\mathcal{T}$. The claim of the Lemma will follow by continuity, once we establish the properties (I) and (II) for the elements of $\mathcal{H}$.

Consider first the map $\eta: U \rightarrow \Delta \cap \mathbb{C}_{\eta\left(J_{U}\right)}$. By the convention we have made, $D(\eta)$ will denote the domain of its three-fold extension $\eta: D(\eta) \rightarrow \mathbb{C}_{\eta\left(J_{U}\right)}$. Set $D \equiv D(\eta) \backslash \eta^{-1} \mathbb{R} \subset$ $\mathbb{C} \backslash \mathbb{R}$, and $\iota: D \hookrightarrow \mathbb{C} \backslash \mathbb{R}$. By Lemma 4.9

$$
\left\|\iota^{\prime}(z)\right\|_{D, \mathbb{C} \backslash \mathbb{R}}<C(s)<1 \text {, where } s=\operatorname{dist}_{D}(z, \partial D) .
$$

On the other hand, $\eta: D \rightarrow \mathbb{C} \backslash \mathbb{R}$ is a local isometry with respect to the hyperbolic metrics, that is $\left\|\eta^{\prime}(z)\right\|_{D, \mathbb{C} \backslash \mathbb{R}}=1$. Thus, $\left\|\eta^{-1}\right\|>C(s)^{-1}>1$, which proves (I) for the map $\eta$.

Let us now establish (II), that is show that for $z \in U$ with $\eta(z) \in \Delta \backslash \Omega,\left\|\eta^{\prime}(z)\right\|>C>1$ for some fixed $C$. Note that $U \cap \mathbb{R}=J_{U}=\left[0, f^{q_{n}-q_{n+1}}(0)\right]$, and $\eta\left(\left[0, f^{q_{n}-q_{n+1}}\right]\right)=\left[q_{n+1}, q_{n}\right]$, which is well inside $\Omega$. This, real a priori bounds, and Koebe Theorem, imply that for some universal $\delta>0$

$$
f^{q_{n+1}}\left(U_{\delta}\left(\left[0, f^{q_{n}-q_{n+1}}(0)\right]\right)\right) \subset \Omega .
$$

Let us now recall the analysis of the shape of the domain $U$ carried out in Ya1. We summarize the relevant results of Ya1 as follows. There exists a universal constant $\epsilon>0$, such that for any point $z \in U \backslash U_{\delta}\left(\left[0, f^{q_{n}-q_{n+1}}(0)\right]\right)$ either $\operatorname{Im} z>\epsilon\left|\left[0, f^{q_{n}-q_{n+1}}(0)\right]\right|$ or $z$ is contained in a possibly empty set $W^{\prime}$ with the following properties. The set $W^{\prime}$ is non-empty only if the iterate $\left.f^{q_{n}}\right|_{\left[f_{n-1}^{\left.q_{n-1}(0), 0\right]}\right.}$ is a sufficiently small perturbation of a parabolic map. In this case $W^{\prime}$ is an $\mathbb{R}$-symmetric topological disk whose inner diameter is commensurable with $\left[0, f^{q_{n}-q_{n+1}}(0)\right]$; and there exists a fundamental domain $C$ for the Douady coordinate 
of $f^{q_{n}}$ such that the univalent image $W=f^{q_{n-1}}\left(W^{\prime}\right)$ is obtained by repeated translation $C_{0}=C, C_{1}, C_{2}, \ldots, C_{k}=C^{\prime}$ of $C$ under $f^{q_{n}}$. The interiors of all the (crescent-shaped) regions $C_{i}$, except for the last one, are disjoint from $C$. The intersection $\left(f^{q_{n-1}}(\partial U) \cap W\right) \cap \mathbb{H}$ is a connected simple curve $\Gamma$ (a "horocycle") obtained by translation of a fundamental arc $\gamma \subset C$ under $f^{q_{n}}$. Note that by compactness of the Epstein class the equatorial annulus which the projections of $\Gamma$ and $-\Gamma$ cut out on the Douady cylinder of $f^{q_{n}}$ has definite modulus.

Now let $z$ be a point of $U$ with $\eta(z)=f^{q_{n+1}}(z) \notin \Omega$. If $z \notin W^{\prime}$, then $\operatorname{dist}_{\mathbb{C} \backslash \mathbb{R}}(z, \partial D)<s_{0}$ with some universal bound $s_{0}$ and we have (II) for $z$. Otherwise, $w=f^{q_{n-1}}(z)$ is contained in one of $C_{i}$. Let $m$ denote the iterate $c^{\prime}=f^{m q_{n}}(c) \in C^{\prime}$. Compactness considerations on the shape of $D\left(f^{q_{n}}\right)$ now imply

$$
\operatorname{dist}_{\mathbb{C} \backslash \mathbb{R}}\left(c^{\prime}, \partial D\left(f^{q_{n}}\right)\right)>s_{1}>0
$$

for some universal $s_{1}$. Hence $\left\|D f^{q_{n}}\left(c^{\prime}\right)\right\|>C>1$. Combining this and property (I) with the chain rule for decomposition $f^{q_{n+1}}=f^{q_{n}} \circ \cdots \circ f^{q_{n}} \circ f^{q_{n-1}}$ we have $\left\|\eta^{\prime}(z)\right\|>C>1$.

The statement (I) is proved in the same way for $\xi$ and $\eta \circ \xi$; (II) is obvious for $\eta \circ \xi$ and is proved in an identical fashion for $\xi$. The statements transfer to limiting renormalization towers by the standard considerations of continuity.

4.3. The structure of the filled Julia set of a limiting renormalization tower. In this section we prove the following theorem:

Theorem 4.12. The filled Julia set $K(\mathcal{T})$ of a limiting renormalization tower $\mathcal{T}$ has no interior, $K(\mathcal{T})=J(\mathcal{T})$. Moreover, repelling periodic orbits of maps in $F(\mathcal{T})$ are dense in $J(\mathcal{T})$.

Lemma 4.13. let $\mathcal{T}$ be a limiting renormalization tower. Then every non-repelling periodic orbit of $\mathcal{T}$ contains the parabolic point of some $\zeta_{n}$ in $\mathcal{T}$.

Proof. Since the claim of the lemma is certainly true for periodic orbits in the real line, let us assume that $z_{1}, \ldots, z_{n}$ is a periodic orbit of a map $h \in F(\mathcal{T})$ disjoint from $\mathbb{R}$. By Lemma 4.11,

$$
\left\|D h^{\circ n}\left(z_{1}\right)\right\|>1
$$

which implies that the orbit $z_{1}, \ldots, z_{n}$ is repelling.

Let us first establish the density of the repelling orbits in $J(\mathcal{T})$,

Lemma 4.14. Repelling periodic orbits of maps in $F(\mathcal{T})$ are dense in $J(\mathcal{T})$. Moreover, for any point $z \in J(\mathcal{T})$ there exists an element $h \in F(\mathcal{T})$ mapping a neighborhood of $z$ onto the domain of $\mathcal{T}$.

Proof. Let $W$ be an open neighborhood of a point $p \in J(\mathcal{T})$. Clearly, there is an element $h \in F(\mathcal{T})$ such that $h(W)$ intersects the Julia set of a holomorphic pair $\mathcal{H} \in \mathcal{T}$. Let us perturb $\mathcal{H}$, if necessary, to a pair $\mathcal{H}^{\prime}$ with an irrational rotation number, so that $W$ still 
intersects $J\left(\mathcal{H}^{\prime}\right)$. By reducing $W$, if necessary, we may assume that $W \cap \mathbb{R}=\emptyset$. Denote by $\mathcal{G}$ a holomorphic extension of the standard pair $\zeta_{\theta, 0}$ quasiconformally conjugate to $\mathcal{H}^{\prime}$. The conjugacy corresponds to $W$ a neighborhood $\tilde{W}$ intersecting the Julia set of the Arnold map $A_{\theta}$. Thus there is an iterate of $A_{\theta}$ mapping $\tilde{W}$ over the whole domain of $\mathcal{G}$. The considerations of convergence imply the existence of an element $h_{W} \in F(\mathcal{T})$ mapping $W$ onto the domain of $\mathcal{T}$. The existense of a repelling periodic point of $\mathcal{T}$ in $W$ follows from Lemma 4.13 .

To prove Theorem 4.12 it remains to show that $K(\mathcal{T})$ has no interior.

Let us call a component $U$ of the interior of $K(\mathcal{T})$ wandering if it is disjoint from its forward images under maps of $F(\mathcal{T})$. A non-wandering component will be called periodic. A modification of the argument used in the above lemma implies that a component of $\stackrel{\circ}{K}(\mathcal{T})$ does not intersect the Julia set of any of the holomorphic pairs $\mathcal{H}$ forming $\mathcal{T}$. This implies that every map $h \in F(\mathcal{T})$ defined on a subset of $U$ is defined on all of $U$. Thus for a periodic component $U$ there exists an $h \in F(\mathcal{T})$ with $h(U)=U$. The disjointness from the Julia sets also implies that $U \cap \mathbb{R}=\emptyset$ for every $U \subset \stackrel{\circ}{K}(\mathcal{T})$.

Lemma 4.15. The filled Julia set of a limiting renormalization tower has no wandering interior components.

Proof. Assume the contrary. Note that since interior components of $K(\mathcal{T})$ do not intersect the real line, $\left.h\right|_{U}$ is univalent for any $U \subset \stackrel{\circ}{K}(\mathcal{T})$ and $h \in F(\mathcal{T})$ defined on $U$. The appropriate case of Sullivan's no wandering domains Theorem can be directly translated to our setting to construct non-trivial quasiconformal deformations of the tower $\mathcal{T}$ entirely supported on the grand orbit of the wandering domain. This contradicts the statement of Theorem 4.5.

We recall the following fundamental principles of dynamics on hyperbolic Riemann surfaces (see e.g. [Lyu1]).

Proposition 4.16. let $h: U \rightarrow U$ be an analytic transform of a hyperbolic Riemann surface. Then one of the following four possibilities holds:

(I) $h$ has an attracting or superattracting fixed point in $U$ to which all points converge;

(II) all orbits tend to infinity;

(III) $h$ is conformally conjugate to an irrational rotation of a disk, a punctured disk or an annulus;

(IV) $h$ is a conformal homeomorphism of finite order.

The next proposition expands on the second possibility:

Proposition 4.17. Let $h$ be an analytic transform of a hyperbolic domain $U \subset \hat{\mathbb{C}}$, continuous up to the boundary of $U$. Suppose that the set of fixed point of $h$ on $\partial U$ is totally disconnected. If the second possibility of Proposition 4.10 occurs, there is a fixed point $\alpha \in \partial U$ such that $h(z) \rightarrow \alpha$ for every $z$. 
The following lemma rules out the existense of periodic components for a limiting renormalization tower, thus completing the proof of Theorem 4.12 .

Lemma 4.18. The filled Julia set of a limiting renormalization tower has no periodic components.

Proof. Assume the contrary. Let $U \subset \stackrel{\circ}{K}(\mathcal{T})$ be a periodic component, and denote by $h$ the element of $F(\mathcal{T})$ fixing $U$. Let us consider the possibilities of Proposition 4.16. The expansion properties of $h$ (Lemma 4.11) rule out the cases (III) and (IV). The case (I) is ruled out by Lemma 4.13. The only remaining possibility is (II). Since $h$ is not the identity map, the fixed points of $h$ in $\partial U$ are isolated, and Proposition 4.17 implies that all orbits in $U$ converge to a fixed point $p$ in $\partial U$. Again applying Lemma 4.13 we see that $p \in \mathbb{R}$ is the parabolic fixed point of one of the commuting pairs forming $\mathcal{T}$.

Denote by $\tilde{U}$ the projection of the domain $U$ onto the repelling Fatou cylinder $C^{R}$ of $p$. The domain $\tilde{U}$ contains the ends \pm of the cylinder. On the other hand, the return map of $C^{R}$ is repelling at the ends (3.1). This implies, that $\tilde{U}$ contains a preimage of the equator $\mathbb{R} / \mathbb{Z}$, and thus $U$ intersects $F(\mathcal{T})^{-1}(\mathbb{R})$. The last statement contradicts the assumption that $U$ is an interior component of $K(\mathcal{T})$.

\subsection{Quasiconformal rigidity of limiting towers: proof of Theorem 4.8.}

Theorem 4.19. A limiting renormalization tower supports no invariant Beltrami differentials on its filled Julia set.

Before giving the proof of the theorem let us state the following

Lemma 4.20 (cf. Lemma 1.8 [Lyu1]). Let $\mathcal{T}$ be a limiting renormalization tower. The group $G$ of homeomorphisms of $K(\mathcal{T})$ which commute with all maps $h \in F(\mathcal{T})$ is totally disconnected.

Proof. Let $\phi \in G$ be a map in the connected component of the identity. Suppose $z_{0} \in K(\mathcal{T})$ is a repelling periodic point with $h\left(z_{0}\right)=z_{0}$ for some $h \in F(\mathcal{T})$. Since the solutions of $h(z)=z$ are isolated, $\phi$ fixes $z_{0}$. The claim now follows from density of repelling cycles, Theorem 4.12 .

Proof of Theorem 4.19. In view of Theorem 4.2 it is enough to prove the statement for standard limiting towers. Let $\mathcal{T}$ be such tower, and let $\mu$ be an invariant Beltrami differential with support in $K(\mathcal{T})$. Let $\sigma_{t}$ denote the complex structure in the plane given by the standard structure $\sigma_{0}$ on $\mathbb{C} \backslash K(\mathcal{T})$, and by $t \mu$ on $K(\mathcal{T})$, where $0 \leq t \leq 1$. Let $\phi_{t}$ be the normalized solution of Beltrami equation $\phi_{t}^{*} \sigma_{0}=\sigma_{t}$. By Theorem 4.5 the towers $\phi_{t} \circ \mathcal{T} \circ \phi_{t}^{-1}$ coincide with $\mathcal{T}$. Thus each map in the continuous family $\phi_{t}$ commutes with all $h \in F(\mathcal{T})$. As $\phi_{0} \equiv \mathrm{Id}$, Lemma 4.20 implies that $\left.\phi_{t}\right|_{K(\mathcal{T})} \equiv \mathrm{Id}$ for all $t$. On the other hand, $\phi_{t}$ is conformal off $K(\mathcal{T})$. Bers' sewing lemma implies that $\phi_{t}$ is conformal in the whole plane, and thus $\phi_{t} \equiv \mathrm{Id}$. This implies that $\mu$ vanishes almost everywhere on $K(\mathcal{T})$. 
For a bi-infinite tower $\mathcal{T}=\left(\mathcal{H}_{k}\right)_{-\infty}^{\infty}$ let $\mathcal{T}^{n}$ denote the truncated tower $\left(\mathcal{H}_{k}\right)_{-n}^{\infty}$. Also denote by $\Omega_{n}$ and $\Delta_{n}$ the domain and range of the pair $\mathcal{H}_{n}$.

Theorem 4.21. Let $\mathcal{T}=\left(\mathcal{H}_{k}\right)_{-\infty}^{\infty}$ be a limiting renormalization bi-infinite tower. Then

$$
\lim _{n \rightarrow-\infty} J\left(\mathcal{T}_{n}\right)=\mathbb{C}
$$

in Hausdorff topology.

Proof. Take any $z \notin \cup_{n<0} J\left(\mathcal{T}_{n}\right)$. Then the orbit $\mathcal{O}_{\mathcal{T}}(z)$ escapes every domain $\Omega_{n}$ for large negative $n$. That is, there exists an infinite sequence of points $z_{n_{k}} \in \mathcal{O}_{\mathcal{T}}(z)$ for $n_{k} \rightarrow-\infty$ such that $z_{n_{k}} \in \Omega_{n_{k}}$ and $\mathcal{H}_{n_{k}}\left(z_{n_{k}}\right) \notin \Omega_{n_{k}}$. Note, that by real a priori bounds and compactness of $\boldsymbol{H}$, the difference $\left|n_{k}-n_{k+1}\right|$ is bounded. By Lemma 4.11 (II) we have $\left\|\mathcal{H}_{n_{k}}^{\prime}\left(z_{n_{k}}\right)\right\|>C>1$ for some universal constant $C$. Moreover, as seen from the proof of the same Lemma, there is an element $h_{n_{k}} \in F\left(\mathcal{T}^{n_{k}-1}\right)$ such that $\operatorname{dist}_{\mathbb{C} \backslash \mathbb{R}}\left(h_{n_{k}}\left(z_{n_{k}}\right), J\left(\mathcal{T}^{n_{k}-1}\right)\right)<$ $s$ for some universal value of $s>0$. Let us arrange the points $\left\{\zeta_{k}=h_{n_{k}}\left(z_{n_{k}}\right)\right\}$ into an infinite orbit $\zeta_{0}=z, \zeta_{1}=g_{0}\left(\zeta_{0}\right), \zeta_{2}=g_{1}\left(\zeta_{1}\right) \ldots$, with $g_{k} \in F(\mathcal{T})$. By Lemma 4.11 (I) we have $\left\|g_{k}^{\prime}\left(\zeta_{k}\right)\right\|>C$. Denote by $\alpha_{k}$ the hyperbolic geodesic in $\mathbb{C} \backslash \mathbb{R}$ of length $l\left(\alpha_{k}\right)<s$ connecting $\zeta_{k}$ to $J\left(\mathcal{T}^{n_{k}-1}\right)$. Let $\alpha_{n_{k}}^{\prime}$ be the connected component of $g_{0}^{-1} \circ \ldots \circ g_{k}^{-1}\left(\alpha_{n_{k}}\right)$ containing $z=\zeta_{0}$. Since the Julia set of a tower is invariant, it is enough to show that $l\left(\alpha_{n_{k}}^{\prime}\right) \rightarrow 0$ as $n_{k} \rightarrow-\infty$. Indeed,

$$
\left\|D g_{k} \circ \cdots \circ g_{0}\right\|>C^{k} \text {. }
$$

By Lemma 4.10 this inequality holds along $\alpha_{n_{k}}^{\prime}$ with $C$ replaced by another universal constant $C_{1}>1$, and hence $\alpha_{n_{k}}^{\prime}$ shrinks to 0 .

Recall, that a measurable line field is a measurable Beltrami differential $u(z)$ with $|u(z)|=$ 1 almost everywhere on the support of $u(z)$. A line field corresponding to a Beltrami differential $\mu$ is $\mu /|\mu|$. We say that $u(z)$ is invariant under a tower $\mathcal{T}$ if for any $h \in F(\mathcal{T})$, $h^{*} u / u$ is a real-valued function. A measurable line field $u(z)$ is univalent on an open set $U$ if $u=h^{*}(d \bar{z} / d z)$ a.e. for a univalent map $h: U \rightarrow \mathbb{C}$.

Proof of Theorem 4.8. Suppose that $\mathcal{T}=\left(\mathcal{H}_{k}\right)_{-\infty}^{\infty}$ is a bi-infinite limiting renormalization tower, and $\mu$ is a nontrivial invariant Beltrami differential of $\mathcal{T}$. Let $u(z)$ denote the corresponding invariant line field. By Theorem $4.19 u(z)$ is not supported in $\cup J\left(\mathcal{T}^{n}\right)$. Let $z$ be a point of almost continuity of $u(z), z \notin J\left(\mathcal{T}^{n}\right)$ for any $n$. Denote again by $z_{n_{k}} \in \Omega_{n_{k}}$ the elements of $\mathcal{O}_{\mathcal{T}}(z)$ with $\mathcal{H}_{n_{k}}\left(z_{n_{k}}\right) \notin \Omega_{n_{k}}$. As seen from the proof of Lemma 4.11, there is an element $h_{n_{k}} \in F\left(\mathcal{T}^{n_{k}-1}\right)$ such that the Euclidean distance from $\zeta_{k}=h_{n_{k}}\left(z_{n_{k}}\right)$ to $\mathbb{R}$ is commensurable with $\left|\Omega_{n_{k}} \cap \mathbb{R}\right|$. Let $\mathcal{T}_{n_{k}}^{\prime}=\Lambda_{n_{k}} \circ \mathcal{T} \circ \Lambda_{n_{k}}^{-1}$ where $\Lambda_{n_{k}}(z)=z /\left|\Omega_{n_{k}} \cap \mathbb{R}\right|$. By compactness of $\boldsymbol{H}$, we may ensure, passing to a subsequence, that elements of $\mathcal{T}_{n_{k}}^{\prime}$ converge to holomorphic pairs $\mathcal{H}_{i}^{\prime}$ forming a bi-infinite tower $\mathcal{T}^{\prime}$. By choosing a further subsequence we may assume that $\Lambda_{n_{k}}^{-1} \zeta_{k} \rightarrow w$, and (see [McM2]) rescaled linefields $u\left(\Lambda_{n_{k}}^{-1}(z)\right) w^{*}$ converge to a measurable linefield $u^{\prime}$, with $h^{*} u^{\prime}=u^{\prime}$ for all $h \in F\left(\mathcal{T}^{\prime}\right)$.

Let $D$ be a small disk around $w$ in $\mathbb{C} \backslash \mathbb{R}$, and denote by $D_{n_{k}}$ its image under the homothety $\Lambda_{n_{k}}$. The diameters of all $D_{n_{k}}$ in the hyperbolic metric of $\mathbb{C} \backslash \mathbb{R}$ are equal. 
Denote by $D_{n_{k}}^{\prime}$ the univalent preimage of $D_{n_{k}}$ by maps of $F(\mathcal{T})$ containing the point $z$. Lemma 4.11 together with Lemma 4.10 imply that Euclidean diameters of $D_{n_{k}}$ shrink to 0 , and $z$ is well inside $D_{n_{k}}$. By McM1, Theorem 5.16] we can choose the linefield $u^{\prime}$ to be univalent on $D$.

By Theorem 4.21 there is an $i$ such that $J\left(\mathcal{H}_{i}^{\prime}\right) \cap D \neq \emptyset$. By Lemma 4.14 the orbit of $D$ by $\mathcal{T}_{i}^{\prime}$ covers all of $\Delta_{i}^{\prime}$. By invariance, this means that $u^{\prime}$ coincides with a locally univalent line field around 0 and $\left(\mathcal{H}_{i}^{\prime}\right)^{2}(0)$ which implies contradictory behaviour of $u^{\prime}$ around $\mathcal{H}_{i}^{\prime}(0)$.

\section{Conclusions}

Proof of Theorems A, B. Due to compactness of $\boldsymbol{H}$, for each bi-infinite string $\rho \in \bar{\Sigma}$ there exits a bi-infinite limiting renormalization tower $\mathcal{T}=\left(\mathcal{H}_{i}\right)_{-\infty}^{\infty}$ with the rotation number $\rho$. In view of Theorem 4.6 such a tower is unique. The element of the set $\mathcal{A}$ corresponding to the string $\rho$ is set to be $\zeta_{\rho}=\mathcal{H}_{0} \cap \mathbb{R}$.

Let $\zeta=\zeta_{\left(\ldots, r_{-n}, \ldots, r_{0}, \ldots, r_{n}, \ldots\right)} \in \mathcal{A}$ be a commuting pair with $r_{0}=\infty$. Let $m$ be the smallest positive integer for which $r_{m}=\infty$ (if such a number does not exist, set $m=\infty$ ). The generalized renormalization $\mathcal{G}$ of such a pair is defined as

$$
\mathcal{G} \zeta=\mathcal{P}_{\tau} \zeta
$$

where the transit map $\tau$ is chosen so that

$$
\rho(\mathcal{G} \zeta)=\left[r_{1}, \ldots, r_{m}\right]
$$

and moreover, when $m$ is finite, $\mathcal{G} \zeta$ is parabolic. By Lemma 4.4 such $\tau$ is unique. The action of $\mathcal{G}$ on $\mathcal{A}$ is injective (Remarks 2.1,3.1); the required invariance properties of $\mathcal{A}$ readily follow from Theorem 4.6.

Let $\zeta \in \mathcal{E}$ be any commuting pair with $\rho(\zeta) \in \mathbb{R} \backslash \mathbb{Q}$, and let $\hat{\zeta}$ be a limit point of the sequence $\mathcal{R}^{n} \zeta$. By compactness of $\boldsymbol{H}, \hat{\zeta}$ is the base pair of a bi-infinite limiting renormalization tower and therefore $\hat{\zeta} \in \mathcal{A}$.

By compactness of $\boldsymbol{H}$, there exists an open neighborhood $G$ of the origin, such that the maximal domains of definition of the elements of $\mathcal{R}^{n} \zeta$ contain $G$ for all large $n$. Now let $\zeta^{\prime} \in \mathcal{A}$ be a commuting pair with $\rho\left(\zeta^{\prime}\right)=\rho(\zeta)$. We will show that

$$
\operatorname{dist}\left(\mathcal{R}^{n} \zeta, \mathcal{R}^{n} \zeta^{\prime}\right) \rightarrow 0
$$

where the distance is measured as the maximum of the distance between the analytic extensions of the elements of the renormalized pairs in $C^{0}$-metric on $G$. Indeed, otherwise there exists a sequence $n_{k} \rightarrow \infty$ and $\epsilon>0$ such that

$$
\operatorname{dist}\left(\mathcal{R}^{n_{k}} \zeta, \mathcal{R}^{n_{k}} \zeta^{\prime}\right)>\epsilon \text {. }
$$

Passing to further subsequence we may ensure that $\mathcal{R}^{n_{k}} \zeta \rightarrow \zeta_{1}, \mathcal{R}^{n_{k}} \zeta^{\prime} \rightarrow \zeta_{2}$ and $\zeta_{1}$ and $\zeta_{2}$ are the base pairs of two limiting renormalization towers $\mathcal{T}_{1}, \mathcal{T}_{2}$ with $\rho\left(\mathcal{T}_{1}\right)=\rho\left(\mathcal{T}_{2}\right)$. The towers $\mathcal{T}_{1}$ and $\mathcal{T}_{2}$ are not homothetic, which contradicts Theorem 4.2 . 
Proof of Theorem $C$. For a parabolic pair $\zeta \in \mathcal{E}$ associate a string $\left(r_{i}\right)_{1}^{\infty}, m \leq \infty$ with $r_{i} \in \mathbb{N} \cup\{\infty\}$. Inductively define the pair $\mathcal{G}^{n+1} \zeta$ as $\mathcal{R}\left(\mathcal{G}^{n} \zeta\right)$ when $\chi\left(\mathcal{G}^{n} \zeta\right) \neq \infty$. If $\mathcal{G}^{n} \zeta$ is a parabolic pair, let $m$ be the smallest positive integer such that $r_{n+m}=\infty$ (if it does not exist, set $m=\infty)$. Set $\mathcal{G}^{n+1} \zeta=\mathcal{P}_{\tau}\left(\mathcal{G}^{n} \zeta\right)$ where the transit map $\tau$ is chosen so that $\rho\left(\mathcal{G}^{n+1} \zeta\right)=\left[r_{n}, r_{n+1}, \ldots, r_{m}\right]$ and, in the case when $m$ is finite, $\mathcal{G}^{n+1} \zeta$ is a parabolic pair (by Lemma 4.4 such $\tau$ is unique).

Consider a sequence of perturbations $\zeta_{k} \rightarrow \zeta$ with $\zeta_{k} \in \mathcal{E}$ and

$$
\rho\left(\zeta_{k}\right)=\left[r_{0}^{k}, r_{1}^{k}, \ldots\right] \in \mathbb{R} \backslash \mathbb{Q} \text {. }
$$

We will also require that $r_{n}^{k} \underset{k \rightarrow \infty}{\longrightarrow} r_{n}$. In view of Lemma 3.4, we may assume by choosing a further subsequence that

$$
\mathcal{R}^{n} \zeta_{k} \underset{k \rightarrow \infty}{\longrightarrow} \mathcal{G}^{n} \zeta .
$$

The existence of uniform real bounds for maps in the Epstein class implies that for $n>N_{0}$ the family $\left\{\mathcal{R}^{n} \zeta_{k}\right\}$ is sequentially pre-compact in $\mathcal{E}$, which means that the sequence $\left\{\mathcal{G}^{n} \zeta\right\}$ is pre-compact as well. Let $\zeta^{*}$ be any limit point of $\left\{\mathcal{G}^{n} \zeta\right\}$. For some choice of $n_{i}$ we have

$$
\mathcal{R}^{n_{i}} \zeta_{k} \rightarrow \zeta^{*} \text {. }
$$

Choosing a "diagonal" subsequence $\zeta_{k_{j}}$ we may ensure that $\mathcal{R}^{n_{i}+L} \zeta_{k_{j}}$ also converge for all $L \in \mathbb{Z}$. Thus $\zeta^{*}$ is a base map of a bi-infinite renormalization limiting tower, and hence $\zeta^{*} \in \mathcal{A}$.

Proof of Theorem D. The pair $\zeta_{0}$ is the base pair of the bi-infinite renormalization tower with rotation number $(\ldots, \infty, \infty, \infty, \ldots)$. The required properties of $\zeta_{0}$ immediately follow from the above considerations.

\section{REFERENCES}

[DD] R.L. Devaney, A. Douady. Homoclinic bifurcations and infinitely many Mandelbrot sets. Preprint.

[Do] A. Douady. Does a Julia set depend continuously on the polynomial? In Complex dynamical systems: The mathematics behind the Mandelbrot set and Julia sets. ed. R.L. Devaney, Proc. of Symposia in Applied Math., Vol 49, Amer. Math. Soc., 1994, pp. 91-138.

[DH] A. Douady, J.H. Hubbard. Etude dynamique des polynômes complexes, I-II. Pub. Math. d'Orsay, 1984.

[dF1] E. de Faria. Proof of universality for critical circle mappings. Thesis, CUNY, 1992.

[dF2] E. de Faria. Asymptotic rigidity of scaling ratios for critical circle mappings. IMS at Stony Brook Preprint \#1996/13.

[dFdM1] E. de Faria and W. de Melo. Rigidity of critical circle mappings I. IMS at Stony Brook Preprint \#1997/16.

[dFdM2] E. de Faria and W. de Melo. Rigidity of critical circle mappings II. IMS at Stony Brook Preprint \#1997/17.

[Ep1] A. Epstein, Towers of finite type complex analytic maps. PhD Thesis, CUNY, 1993.

[Ep2] A. Epstein, Counterexamples to the quadratic mating conjecture. Manuscript in preparation.

[EKT] A. Epstein, L. Keen, C. Tresser. The set of maps $F_{a, b}: x \mapsto x+a+\frac{b}{2 \pi} \sin (2 \pi x)$ with any given rotation interval is contractible. Commun. Math. Phys. 173, 313-333, 1995.

[EE] J.-P. Eckmann and H. Epstein. On the existence of fixed points of the composition operator for circle maps. Commun. Math. Phys. 107 (1986), 213-231. 
[FKS] M. Feigenbaum, L. Kadanoff, and S. Shenker. Quasi-periodicity in dissipative systems. A renormalization group analysis. Physica 5D (1982), 370-386.

[He] M. Herman. Conjugaison quasi-symmetrique des homeomorphismes analytiques du cercle a des rotations. Manuscript.

[Hin] B. Hinkle. Parabolic limits of renormalization. IMS at Stony Brook Preprint \#1997/7

[Keen] L. Keen. Dynamics of holomorphic self-maps of $\mathbb{C}^{*}$. In Holomorphic functions and moduli I (ed. D. Drasin et al.) Springer-verlag, New York, 1988.

[Lan1] O.E. Lanford. Renormalization group methods for critical circle mappings with general rotation number, VIIIth International Congress on Mathematical Physics (Marseille,1986), World Sci. Publishing, Singapore, 532-536, (1987).

[Lan2] O.E. Lanford. Renormalization group methods for critical circle mappings. Nonlinear evolution and chaotic phenomena, NATO adv. Sci. Inst. Ser. B:Phys.,176, Plenum, New York, 25-36, (1988).

[Lyu1] M. Lyubich. The dynamics of rational transforms: the topological picture. Russ. Math. Surveys 41(1986), 35-95.

[Lyu2] M. Lyubich. Renormalization ideas in conformal dynamics. Cambridge Seminar "Current Developments in Math.", May 1995. International Press, 1995. Cambridge, MA, 155-184.

[Lyu3] M. Lyubich. Feigenbaum-Coullet-Tresser Universality and Milnor's Hairiness Conjecture. Preprint IHES/M/96/61.

[Lyu4] M. Lyubich. Almost every real quadratic map is either regular or stochastic. IMS at Stony Brook Preprint \#1997/8.

[LY] M. Lyubich and M.Yampolsky. Dynamics of quadratic polynomials: complex bounds for real maps. MSRI Preprint 034-95, 1995, to appear in Ann. Inst. Fourier.

[McM1] C. McMullen. Complex dynamics and renormalization. Annals of Math. Studies, v.135, Princeton Univ. Press, 1994.

[McM2] C. McMullen. Renormalization and 3-manifolds which fiber over the circle. Annals of Math. Studies, Princeton University Press, 1996.

[MvS] W. de Melo \& S. van Strien. One dimensional dynamics. Springer-Verlag, 1993.

[ORSS] S. Ostlund, D. Rand, J. Sethna, E. Siggia, Universal properties of the transition from quasiperiodicity to chaos in dissipative systems. Physica, $\mathbf{8 D}(1983), 303-342$.

[Sul1] D.Sullivan. Quasiconformal homeomorphisms and dynamics, topology and geometry. Proc. ICM86, Berkeley, v. II, 1216-1228.

[Sul2] D.Sullivan. Bounds, quadratic differentials, and renormalization conjectures. AMS Centennial Publications. 2: Mathematics into Twenty-first Century (1992).

[Sh] M. Shishikura. The parabolic bifurcation of rational maps. Colóquo 19 Brasileiro de Matemática, IMPA.

[Sw1] G.Światek. Rational rotation numbers for maps of the circle. Commun. Math. Phys. 119, 109-128 (1988).

[Ya1] M. Yampolsky. Complex bounds for renormalization of critical circle maps, to appear in Erg. Th. E Dyn. Systems. An earlier version available as IMS at Stony Brook Preprint 1995/12.

[Yoc] J.-C. Yoccoz, Il n'ya pas de contre-example de Denjoy analytique, C.R. Acad. Sci. Paris 298(1984) série I, 141-144. 